\title{
Auxiliary Linear Problem, Difference Fay Identities and Dispersionless Limit of Pfaff-Toda Hierarchy
}

\author{
Kanehisa TAKASAKI \\ Graduate School of Human and Environmental Studies, Kyoto University, \\ Yoshida, Sakyo, Kyoto, 606-8501, Japan \\ E-mail: takasaki@math.h.kyoto-u.ac.jp \\ URL: http://www.math.h.kyoto-u.ac.jp/ takasaki/
}

Received August 27, 2009, in final form December 15, 2009; Published online December 19, 2009 doi:10.3842/SIGMA.2009.109

\begin{abstract}
Recently the study of Fay-type identities revealed some new features of the DKP hierarchy (also known as "the coupled KP hierarchy" and "the Pfaff lattice"). Those results are now extended to a Toda version of the DKP hierarchy (tentatively called "the Pfaff-Toda hierarchy"). Firstly, an auxiliary linear problem of this hierarchy is constructed. Unlike the case of the DKP hierarchy, building blocks of the auxiliary linear problem are difference operators. A set of evolution equations for dressing operators of the wave functions are also obtained. Secondly, a system of Fay-like identities (difference Fay identities) are derived. They give a generating functional expression of auxiliary linear equations. Thirdly, these difference Fay identities have well defined dispersionless limit (dispersionless Hirota equations). As in the case of the DKP hierarchy, an elliptic curve is hidden in these dispersionless Hirota equations. This curve is a kind of spectral curve, whose defining equation is identified with the characteristic equation of a subset of all auxiliary linear equations. The other auxiliary linear equations are related to quasi-classical deformations of this elliptic spectral curve.
\end{abstract}

Key words: integrable hierarchy; auxiliary linear problem; Fay-like identity; dispersionless limit; spectral curve; quasi-classical deformation

2000 Mathematics Subject Classification: 35Q58; 37K10

\section{Introduction}

This paper is a sequel of the study on Fay-type identities of integrable hierarchies, in particular the DKP hierarchy [1]. The DKP hierarchy is a variant of the KP hierarchy and obtained as a subsystem of Jimbo and Miwa's hierarchy of the $D_{\infty}^{\prime}$ type $[2,3]$. The same hierarchy was rediscovered later on as "the coupled KP hierarchy" [4] and "the Pfaff lattice" [5, 6, 7], and has been studied from a variety of points of view $[8,9,10,11,12,13,14,15,16]$. The term "Pfaff" stems from the fact that Pfaffians play a role in many aspects of this system. The previous study [1] revealed some new features of this relatively less known integrable hierarchy. In this paper, we extend those results to a Toda version of the DKP hierarchy.

The integrable hierarchy in question is a slight modification of the system proposed by Willox $[17,18]$ as an extension of the Jimbo-Miwa $D_{\infty}^{\prime}$ hierarchy. We call this system, tentatively, "the Pfaff-Toda hierarchy" (as an abbreviation of the "Pfaffian" or "Pfaffianized" Toda hierarchy). Following the construction of Jimbo and Miwa, Willox started from a fermionic definition of the tau function, and derived this hierarchy in a bilinear form. The lowest level of this hierarchy contains a $2+2 D$ ( 2 continuous and 2 discrete $)$ extension

$$
\begin{gathered}
\frac{1}{2} D_{x} D_{y} \tau(s, r, x, y) \cdot \tau(s, r, x, y)+\tau(s-1, r, x, y) \tau(s+1, r, x, y) \\
-\tau(s, r-1, x, y) \tau(s, r+1, x, y)=0
\end{gathered}
$$


of the usual $2+1 D$ Toda equation and an additional $2+2 D$ equation

$$
D_{x} \tau(s, r, x, y) \cdot \tau(s+1, r-1, x, y)+D_{y} \tau(s, r-1, x, y) \cdot \tau(s+1, r, x, y)=0
$$

(see the papers of Santini et al. [19], Hu et al. [20] and Gilson and Nimmo [21] for some other sources of these equations). Willox further presented an auxiliary linear problem for these lowest equations, but extending it to the full hierarchy was an open problem. We first address this issue, then turn to issues of Fay-like identities and dispersionless limit.

As we show in this paper, the Pfaff-Toda hierarchy is indeed a mixture of the DKP and Toda hierarchies. Firstly, we can formulate an auxiliary linear problem as a two-component system like that of the DKP hierarchy [1], but building blocks therein are difference (rather than differential) operators as used for the Toda hierarchy. Secondly, the differential Fay identities of the DKP hierarchy are replaced by "difference Fay identities" analogous to those of the Toda hierarchy $[22,23]$. Lastly, those difference Fay identities have well defined dispersionless limit to the so called "dispersionless Hirota equations". These equations resemble the dispersionless Hirota equations of the Toda hierarchy [22, 24, 25, 26], but exhibits a more complicated structure parallel to the dispersionless Hirota equations of the DKP hierarchy [1].

Among these rich contents, a particularly remarkable outcome is the fact that an elliptic curve is hidden in the dispersionless Hirota equations. A similar elliptic curve was also encountered in the dispersionless Hirota equations of the DKP hierarchy [1], but its true meaning remained to be clarified. This puzzle was partly resolved by Kodama and Pierce [27]. They interpreted the curve as an analogue of the "spectral curve" of the dispersionless 1D Toda lattice. We can now give a more definite answer to this issue. Namely, these curves are defined by the characteristic equations of a subset of the full auxiliary linear equations, hence may be literally interpreted as spectral curves. The other auxiliary linear equations are related to "quasi-classical deformations" [28, 29] of these curves.

This paper is organized as follows. In Section 2, we formulate the Pfaff-Toda hierarchy as a bilinear equation for the tau function. This bilinear equation is actually a generating functional expression of an infinite number of Hirota equations. In Section 3, we present a full system of auxiliary linear equations that contains Willox's auxiliary linear equations. A system of evolution equations for "dressing operators" of the wave functions are also obtained. The dressing operators are difference operators in a direction ( $s$-direction) of the $2 \mathrm{D}$ lattice; another direction ( $r$-direction) plays the role of a discrete time variable. Section 4 deals with the difference Fay identities. These Fay-like identities are derived from the bilinear equation of Section 2 by specializing the values of free variables. We show that they are auxiliary linear equations in disguise, namely, they give a generating functional expression of the auxiliary linear equations of Section 3. Section 5 is devoted to the issues of dispersionless limit. The dispersionless Hirota equations are derived from the differential Fay identities as a kind of "quasi-classical limit". After rewriting these dispsersionless Hirota equations, we find an elliptic curve hidden therein, and identify a set of auxiliary linear equations for which the curve can be interpreted as a spectral curve.

\section{$2 \quad$ Bilinear equations}

The Pfaff-Toda hierarchy has two discrete variables $s, r \in \mathbf{Z}$ and two sets of continuous variables $\boldsymbol{t}=\left(t_{1}, t_{2}, \ldots\right), \overline{\boldsymbol{t}}=\left(\bar{t}_{1}, \bar{t}_{2}, \ldots\right)$. In this section, we present this hierarchy in a bilinear form, which comprises various bilinear equations for the tau function $\tau=\tau(s, r, \boldsymbol{t}, \overline{\boldsymbol{t}})$. In the following consideration, we shall frequently use shortened notations such as $\tau(s, r)$ for $\tau(s, r, \boldsymbol{t}, \overline{\boldsymbol{t}})$ to save spaces. 


\subsection{Bilinear equation of contour integral type}

The most fundamental bilinear equation is the equation

$$
\begin{aligned}
& \oint \frac{d z}{2 \pi i} z^{s^{\prime}+r^{\prime}-s-r} e^{\xi\left(\boldsymbol{t}^{\prime}-\boldsymbol{t}, z\right)} \tau\left(s^{\prime}, r^{\prime}, \boldsymbol{t}^{\prime}-\left[z^{-1}\right], \overline{\boldsymbol{t}}^{\prime}\right) \tau\left(s, r, \boldsymbol{t}+\left[z^{-1}\right], \overline{\boldsymbol{t}}\right) \\
& \quad+\oint \frac{d z}{2 \pi i} z^{s+r-s^{\prime}-r^{\prime}-4} e^{\xi\left(\boldsymbol{t}-\boldsymbol{t}^{\prime}, z\right)} \tau\left(s^{\prime}+1, r^{\prime}+1, \boldsymbol{t}^{\prime}+\left[z^{-1}\right], \overline{\boldsymbol{t}}^{\prime}\right) \tau\left(s-1, r-1, \boldsymbol{t}-\left[z^{-1}\right], \overline{\boldsymbol{t}}\right) \\
& =\oint \frac{d z}{2 \pi i} z^{s^{\prime}-r^{\prime}-s+r} e^{\xi\left(\overline{\boldsymbol{t}}^{\prime}-\overline{\boldsymbol{t}}, z^{-1}\right)} \tau\left(s^{\prime}+1, r^{\prime}, \boldsymbol{t}^{\prime}, \overline{\boldsymbol{t}}^{\prime}-[z]\right) \tau(s-1, r, \boldsymbol{t}, \overline{\boldsymbol{t}}+[z]) \\
& \quad+\oint \frac{d z}{2 \pi i} z^{s-r-s^{\prime}+r^{\prime}} e^{\xi\left(\overline{\boldsymbol{t}}-\overline{\boldsymbol{t}}^{\prime}, z^{-1}\right)} \tau\left(s^{\prime}, r^{\prime}+1, \boldsymbol{t}^{\prime}, \overline{\boldsymbol{t}}^{\prime}+[z]\right) \tau(s, r-1, \boldsymbol{t}, \overline{\boldsymbol{t}}-[z])
\end{aligned}
$$

that is understood to hold for arbitrary values of $(s, r, \boldsymbol{t}, \overline{\boldsymbol{t}})$ and $\left(s^{\prime}, r^{\prime}, \boldsymbol{t}^{\prime}, \overline{\boldsymbol{t}}^{\prime}\right)$. This equation is a modification of the bilinear equation derived by Willox $[17,18]$ in a fermionic construction of the tau function (see Section 2.3 below). Note that we have used the standard notations

$$
[z]=\left(z, \frac{z^{2}}{2}, \frac{z^{3}}{3}, \ldots\right), \quad \xi(\boldsymbol{t}, z)=\sum_{k=1}^{\infty} t_{k} z^{k},
$$

and both hand sides of the bilinear equation are contour integrals along simple closed cycles $C_{\infty}$ (for integrals on the left hand side) and $C_{0}$ (for integrals on the right hand side) that encircle the points $z=\infty$ and $z=0$. Actually, since these integrals simply extract the coefficient of $z^{-1}$ from Laurent expansion at those points, we can redefine these integrals as a genuine linear map from Laurent series to constants:

$$
\oint \frac{d z}{2 \pi i} \sum_{n=-\infty}^{\infty} a_{n} z^{n}=a_{-1} .
$$

As we show below, this bilinear equation is a generating functional expression of an infinite number of Hirota equations.

In some cases, it is more convenient to shift $s$ and $r$ as $s \rightarrow s+1$ and $r \rightarrow r+1$. The outcome is the equation

$$
\begin{aligned}
\oint \frac{d z}{2 \pi i} z^{s^{\prime}+r^{\prime}-s-r-2} e^{\xi\left(\boldsymbol{t}^{\prime}-\boldsymbol{t}, z\right)} \tau\left(s^{\prime}, r^{\prime}, \boldsymbol{t}^{\prime}-\left[z^{-1}\right], \overline{\boldsymbol{t}}^{\prime}\right) \tau\left(s+1, r+1, \boldsymbol{t}+\left[z^{-1}\right], \overline{\boldsymbol{t}}\right) \\
\quad+\oint \frac{d z}{2 \pi i} z^{s+r-s^{\prime}-r^{\prime}-2} e^{\xi\left(\boldsymbol{t}-\boldsymbol{t}^{\prime}, z\right)} \tau\left(s^{\prime}+1, r^{\prime}+1, \boldsymbol{t}^{\prime}+\left[z^{-1}\right], \overline{\boldsymbol{t}}^{\prime}\right) \tau\left(s, r, \boldsymbol{t}-\left[z^{-1}\right], \overline{\boldsymbol{t}}\right) \\
=\oint \frac{d z}{2 \pi i} z^{s^{\prime}-r^{\prime}-s+r} e^{\xi\left(\overline{\boldsymbol{t}}^{\prime}-\overline{\boldsymbol{t}}, z^{-1}\right)} \tau\left(s^{\prime}+1, r^{\prime}, \boldsymbol{t}^{\prime}, \overline{\boldsymbol{t}}^{\prime}-[z]\right) \tau(s, r+1, \boldsymbol{t}, \overline{\boldsymbol{t}}+[z]) \\
\quad+\oint \frac{d z}{2 \pi i} z^{s-r-s^{\prime}+r^{\prime}} e^{\xi\left(\overline{\boldsymbol{t}}-\overline{\boldsymbol{t}}^{\prime}, z^{-1}\right)} \tau\left(s^{\prime}, r^{\prime}+1, \boldsymbol{t}^{\prime}, \overline{\boldsymbol{t}}^{\prime}+[z]\right) \tau(s+1, r, \boldsymbol{t}, \overline{\boldsymbol{t}}-[z]) .
\end{aligned}
$$

By changing variables as $z \rightarrow z^{-1}$ on the right hand side, this equation can be converted to a more symmetric form as

$$
\begin{aligned}
\oint \frac{d z}{2 \pi i} z^{s^{\prime}+r^{\prime}-s-r-2} e^{\xi\left(\boldsymbol{t}^{\prime}-\boldsymbol{t}, z\right)} \tau\left(s^{\prime}, r^{\prime}, \boldsymbol{t}^{\prime}-\left[z^{-1}\right], \overline{\boldsymbol{t}}^{\prime}\right) \tau\left(s+1, r+1, \boldsymbol{t}+\left[z^{-1}\right], \overline{\boldsymbol{t}}\right) \\
\quad+\oint \frac{d z}{2 \pi i} z^{s+r-s^{\prime}-r^{\prime}-2} e^{\xi\left(\boldsymbol{t}-\boldsymbol{t}^{\prime}, z\right)} \tau\left(s^{\prime}+1, r^{\prime}+1, \boldsymbol{t}^{\prime}+\left[z^{-1}\right], \overline{\boldsymbol{t}}^{\prime}\right) \tau\left(s, r, \boldsymbol{t}-\left[z^{-1}\right], \overline{\boldsymbol{t}}\right) \\
=\oint \frac{d z}{2 \pi i} z^{-s^{\prime}+r^{\prime}+s-r-2} e^{\xi\left(\overline{\boldsymbol{t}}^{\prime}-\overline{\boldsymbol{t}}, z\right)} \tau\left(s^{\prime}+1, r^{\prime}, \boldsymbol{t}^{\prime}, \overline{\boldsymbol{t}}^{\prime}-\left[z^{-1}\right]\right) \tau\left(s, r+1, \boldsymbol{t}, \overline{\boldsymbol{t}}+\left[z^{-1}\right]\right) \\
\quad+\oint \frac{d z}{2 \pi i} z^{-s+r+s^{\prime}-r^{\prime}-2} e^{\xi\left(\overline{\boldsymbol{t}}-\overline{\boldsymbol{t}}^{\prime}, z\right)} \tau\left(s^{\prime}, r^{\prime}+1, \boldsymbol{t}^{\prime}, \overline{\boldsymbol{t}}^{\prime}+\left[z^{-1}\right]\right) \tau\left(s+1, r, \boldsymbol{t}, \overline{\boldsymbol{t}}-\left[z^{-1}\right]\right),
\end{aligned}
$$

though we shall not pursue this line further. 


\subsection{Hirota equations}

Following the standard procedure, we now introduce arbitrary constants

$$
\boldsymbol{a}=\left(a_{1}, a_{2}, \ldots\right), \quad \overline{\boldsymbol{a}}=\left(\bar{a}_{1}, \bar{a}_{2}, \ldots\right)
$$

and shift the continuous variables $\boldsymbol{t}, \boldsymbol{t}^{\prime}, \overline{\boldsymbol{t}}, \overline{\boldsymbol{t}}^{\prime}$ in the bilinear equation (2.1) as

$$
t^{\prime} \rightarrow t-a, \quad \bar{t}^{\prime} \rightarrow \bar{t}-\bar{a}, \quad t \rightarrow t+a, \quad \bar{t} \rightarrow \bar{t}+\bar{a} .
$$

The bilinear equation thereby takes such a form as

$$
\begin{aligned}
& \oint \frac{d z}{2 \pi i} z^{s^{\prime}+r^{\prime}-s-r} e^{-2 \xi(\boldsymbol{a}, z)} \tau\left(s^{\prime}, r^{\prime}, \boldsymbol{t}-\boldsymbol{a}-\left[z^{-1}\right], \overline{\boldsymbol{t}}-\overline{\boldsymbol{a}}\right) \tau\left(s, r, \boldsymbol{t}+\boldsymbol{a}+\left[z^{-1}\right], \overline{\boldsymbol{t}}+\overline{\boldsymbol{a}}\right) \\
& +\oint \frac{d z}{2 \pi i} z^{s+r-s^{\prime}-r^{\prime}-4} e^{2 \xi(\boldsymbol{a}, z)} \tau\left(s^{\prime}+1, r^{\prime}+1, \boldsymbol{t}-\boldsymbol{a}+\left[z^{-1}\right], \overline{\boldsymbol{t}}-\overline{\boldsymbol{a}}\right) \\
& \quad \times \tau\left(s-1, r-1, \boldsymbol{t}+\boldsymbol{a}-\left[z^{-1}\right], \overline{\boldsymbol{t}}+\overline{\boldsymbol{a}}\right) \\
& =\oint \frac{d z}{2 \pi i} z^{s^{\prime}-r^{\prime}-s+r} e^{-2 \xi\left(\overline{\boldsymbol{a}}, z^{-1}\right)} \tau\left(s^{\prime}+1, r^{\prime}, \boldsymbol{t}-\boldsymbol{a}, \overline{\boldsymbol{t}}-\overline{\boldsymbol{a}}-[z]\right) \tau(s-1, r, \boldsymbol{t}+\boldsymbol{a}, \overline{\boldsymbol{t}}+\overline{\boldsymbol{a}}+[z]) \\
& +\oint \frac{d z}{2 \pi i} z^{s-r-s^{\prime}+r^{\prime}} e^{2 \xi\left(\overline{\boldsymbol{a}}, z^{-1}\right)} \tau\left(s^{\prime}, r^{\prime}+1, \boldsymbol{t}-\boldsymbol{a}, \overline{\boldsymbol{t}}-\overline{\boldsymbol{a}}+[z]\right) \tau(s, r-1, \boldsymbol{t}+\boldsymbol{a}, \overline{\boldsymbol{t}}+\overline{\boldsymbol{a}}-[z]) .
\end{aligned}
$$

With the aid of Hirota's notations

$$
D_{t_{n}} f \cdot g=\partial_{t_{n}} f \cdot g-f \cdot \partial_{t_{n}} g, \quad D_{\bar{t}_{n}} f \cdot g=\partial_{\bar{t}_{n}} f \cdot g-f \cdot \partial_{\bar{t}_{n}} g,
$$

the product of two shifted tau functions in each term of this equation can be expressed as

$$
\begin{gathered}
\tau\left(s^{\prime}, r^{\prime}, \boldsymbol{t}-\boldsymbol{a}-\left[z^{-1}\right], \overline{\boldsymbol{t}}-\overline{\boldsymbol{a}}\right) \tau\left(s, r, \boldsymbol{t}+\boldsymbol{a}+\left[z^{-1}\right], \overline{\boldsymbol{t}}+\overline{\boldsymbol{a}}\right) \\
=e^{\xi\left(\tilde{D}_{t}, z^{-1}\right)} e^{\left\langle\boldsymbol{a}, D_{t}\right\rangle+\left\langle\overline{\boldsymbol{a}}, D_{\bar{t}}\right\rangle} \tau(s, r, \boldsymbol{t}, \overline{\boldsymbol{t}}) \cdot \tau\left(s^{\prime}, r^{\prime}, \boldsymbol{t}, \overline{\boldsymbol{t}}\right),
\end{gathered}
$$

etc., where $D_{t}$ and $D_{\bar{t}}$ denote the arrays

$$
D_{t}=\left(D_{t_{1}}, D_{t_{2}}, \ldots, D_{t_{n}}, \ldots\right), \quad D_{\bar{t}}=\left(D_{\bar{t}_{1}}, D_{\bar{t}_{2}}, \ldots, D_{\bar{t}_{n}}, \ldots\right)
$$

of Hirota bilinear operators, $\tilde{D}_{t}$ and $\tilde{D}_{\bar{t}}$ their variants

$$
\tilde{D}_{t}=\left(D_{t_{1}}, \frac{1}{2} D_{t_{2}}, \ldots, \frac{1}{n} D_{t_{n}}, \ldots\right), \quad \tilde{D}_{\bar{t}}=\left(D_{\bar{t}_{1}}, \frac{1}{2} D_{\bar{t}_{2}}, \ldots, \frac{1}{n} D_{\bar{t}_{n}}, \ldots\right),
$$

and $\left\langle\boldsymbol{a}, D_{t}\right\rangle$ and $\left\langle\overline{\boldsymbol{a}}, D_{\bar{t}}\right\rangle$ their linear combinations

$$
\left\langle\boldsymbol{a}, D_{t}\right\rangle=\sum_{n=1}^{\infty} a_{n} D_{t_{n}}, \quad\left\langle\overline{\boldsymbol{a}}, D_{\bar{t}}\right\rangle=\sum_{n=1}^{\infty} \bar{a}_{n} D_{\bar{t}_{n}} .
$$

Let us introduce the functions $h_{n}(\boldsymbol{t}), n \geq 0$, defined by the generating function

$$
\sum_{n=0}^{\infty} h_{n}(\boldsymbol{t}) z^{n}=e^{\xi(\boldsymbol{t}, z)} .
$$

The first few terms read

$$
h_{0}(\boldsymbol{t})=1, \quad h_{1}(\boldsymbol{t})=t_{1}, \quad h_{2}(\boldsymbol{t})=\frac{t_{1}^{2}}{2}+t_{2}, \quad h_{3}(\boldsymbol{t})=\frac{t_{1}^{3}}{6}+t_{1} t_{2}+t_{3},
$$


The prefactors $e^{ \pm 2 \xi(\boldsymbol{a}, z)}$, etc., can be thereby expanded as

$$
e^{ \pm 2 \xi(\boldsymbol{a}, z)}=\sum_{n=0}^{\infty} h_{n}( \pm 2 \boldsymbol{a}) z^{n}, \quad e^{ \pm 2 \xi\left(\overline{\boldsymbol{a}}, z^{-1}\right)}=\sum_{n=0}^{\infty} h_{n}( \pm 2 \overline{\boldsymbol{a}}) z^{-n} .
$$

Similarly, the exponential operators $e^{ \pm \xi\left(\tilde{D}_{t}, z^{-1}\right)}$, etc., can be expanded as

$$
e^{ \pm \xi\left(\tilde{D}_{t}, z^{-1}\right)}=\sum_{n=0}^{\infty} h_{n}\left(\tilde{D}_{t}\right) z^{-n}, \quad e^{ \pm \xi\left(\tilde{D}_{\bar{t}}, z\right)}=\sum_{n=0}^{\infty} h_{n}\left(\tilde{D}_{\bar{t}}\right) z^{n} .
$$

The bilinear equation thus turns into the Hirota form

$$
\begin{aligned}
\sum_{n=0}^{\infty} h_{n}( & -2 \boldsymbol{a}) h_{n+s^{\prime}+r^{\prime}-s-r+1}\left(\tilde{D}_{t}\right) e^{\left\langle\boldsymbol{a}, D_{t}\right\rangle+\left\langle\overline{\boldsymbol{a}}, D_{\bar{t}}\right\rangle} \tau(s, r) \cdot \tau\left(s^{\prime}, r^{\prime}\right) \\
& +\sum_{n=0}^{\infty} h_{n}(2 \boldsymbol{a}) h_{n+s+r-s^{\prime}-r^{\prime}-3}\left(-\tilde{D}_{t}\right) e^{\left\langle\boldsymbol{a}, D_{t}\right\rangle+\left\langle\overline{\boldsymbol{a}}, D_{\bar{t}}\right\rangle} \tau(s-1, r-1) \cdot \tau\left(s^{\prime}+1, r^{\prime}+1\right) \\
= & \sum_{n=0}^{\infty} h_{n}(-2 \overline{\boldsymbol{a}}) h_{n-s^{\prime}+r^{\prime}+s-r-1}\left(\tilde{D}_{\bar{t})} e^{\left\langle\boldsymbol{a}, D_{t}\right\rangle+\left\langle\overline{\boldsymbol{a}}, D_{\bar{t}}\right\rangle} \tau(s-1, r) \cdot \tau\left(s^{\prime}+1, r^{\prime}\right)\right. \\
& +\sum_{n=0}^{\infty} h_{n}(2 \overline{\boldsymbol{a}}) h_{n-s+r+s^{\prime}-r^{\prime}-1}\left(-\tilde{D}_{\bar{t}}\right) e^{\left\langle\boldsymbol{a}, D_{t}\right\rangle+\left\langle\overline{\boldsymbol{a}}, D_{\bar{t}}\right\rangle} \tau(s, r-1) \cdot \tau\left(s^{\prime}, r^{\prime}+1\right) .
\end{aligned}
$$

The last equation is still a generating functional expression, from which one can derive an infinite number of equations by Taylor expansion of both hand sides at $\boldsymbol{a}=\mathbf{0}$ and $\overline{\boldsymbol{a}}=\mathbf{0}$. For example, the linear part of the expansion give the equations

$$
\begin{aligned}
\left(-2 h_{n+s^{\prime}+r^{\prime}-s-r+1}\left(\tilde{D}_{t}\right)+h_{s^{\prime}+r^{\prime}-s-r+1}\left(\tilde{D}_{t}\right) D_{t_{n}}\right) \tau(s, r) \cdot \tau\left(s^{\prime}, r^{\prime}\right) \\
\quad+\left(2 h_{n+s+r-s^{\prime}-r^{\prime}-3}\left(-\tilde{D}_{t}\right)+h_{s+r-s^{\prime}-r^{\prime}-3}\left(-\tilde{D}_{t}\right) D_{t_{n}}\right) \tau(s-1, r-1) \cdot \tau\left(s^{\prime}+1, r^{\prime}+1\right) \\
=h_{-s^{\prime}+r^{\prime}+s-r-1}\left(\tilde{D}_{\bar{t}}\right) D_{t_{n}} \tau(s-1, r) \cdot \tau\left(s^{\prime}+1, r^{\prime}\right) \\
\quad+h_{-s+r+s^{\prime}-r^{\prime}-1}\left(-\tilde{D}_{\bar{t}}\right) D_{t_{n}} \tau(s, r-1) \cdot \tau\left(s^{\prime}, r^{\prime}+1\right)
\end{aligned}
$$

and

$$
\begin{aligned}
h_{s^{\prime}+r^{\prime}-s-r+1}\left(\tilde{D}_{t}\right) D_{\bar{t}_{n}} \tau(s, r) \cdot \tau\left(s^{\prime}, r^{\prime}\right) \\
\quad+h_{s+r-s^{\prime}-r^{\prime}-3}\left(-\tilde{D}_{t}\right) D_{\bar{t}_{n}} \tau(s-1, r-1) \cdot \tau\left(s^{\prime}+1, r^{\prime}+1\right) \\
=\left(-2 h_{n-s^{\prime}+r^{\prime}+s-r-1}\left(\tilde{D}_{\bar{t}}\right)+h_{-s^{\prime}+r^{\prime}+s-r-1}\left(\tilde{D}_{\bar{t}_{n}}\right) D_{\bar{t}_{n}}\right) \tau(s-1, r) \cdot \tau\left(s^{\prime}+1, r^{\prime}\right) \\
\quad+\left(2 h_{n-s+r+s^{\prime}-r^{\prime}-1}\left(-\tilde{D}_{\bar{t}}\right)+h_{-s+r+s^{\prime}-r^{\prime}-1}\left(-\tilde{D}_{\bar{t}}\right) D_{\bar{t}_{n}}\right) \tau(s, r-1) \cdot \tau\left(s^{\prime}, r^{\prime}+1\right)
\end{aligned}
$$

for $n=0,1, \ldots$ In particular, the special case of (2.4) where $s^{\prime}=s, r^{\prime}=r$ and $n=1$ gives the equation

$$
\frac{1}{2} D_{t_{1}} D_{\bar{t}_{1}} \tau(s, r) \cdot \tau(s, r)+\tau(s-1, r) \tau(s+1, r)-\tau(s, r-1) \tau(s, r+1)=0
$$

Moreover, specializing (2.3) to $\boldsymbol{a}=\overline{\boldsymbol{a}}=\mathbf{0}, s^{\prime}=s+1$ and $r^{\prime}=r-1$ yields the equation

$$
D_{t_{1}} \tau(s, r) \cdot \tau(s+1, r-1)+D_{\bar{t}_{1}} \tau(s, r-1) \cdot \tau(s+1, r)=0 .
$$

These equations give the lowest part of the whole Hirota equations. 


\subsection{Fermionic formula of tau functions}

Solutions of these bilinear equations are given by ground state expectation values of operators on the Fock space of 2D complex free fermions.

Let us recall basic constituents of the fermion system. $\psi_{j}, \psi_{j}^{*}(j \in \mathbf{Z})$ denote the Fourier modes of fermion fields

$$
\psi(z)=\sum_{j=-\infty}^{\infty} \psi_{j} z^{j}, \quad \psi^{*}(z)=\sum_{j=-\infty}^{\infty} \psi_{j}^{*} z^{-j-1} .
$$

They obey the anti-commutation relations

$$
\left[\psi_{j}, \psi_{k}^{*}\right]_{+}=\delta_{j k}, \quad\left[\psi_{j}, \psi_{k}\right]_{+}=\left[\psi_{j}^{*}, \psi_{k}^{*}\right]_{+}=0 .
$$

$|0\rangle$ and $\langle 0|$ denote the vacuum states characterized by the annihilation conditions

$$
\begin{aligned}
& \langle 0| \psi_{j}=0 \quad \text { for } j \geq 0, \quad \psi_{j}|0\rangle=0 \quad \text { for } j<0, \\
& \langle 0| \psi_{j}^{*}=0 \quad \text { for } j<0, \quad \psi_{j}^{*}|0\rangle=0 \quad \text { for } j \geq 0 \text {. }
\end{aligned}
$$

The Fock space and its dual space are generated these vacuum states, and decomposed to eigenspaces of the charge operator

$$
H_{0}=\sum_{j=-\infty}^{\infty}: \psi_{j} \psi_{j}^{*}: \quad \text { (normal ordering). }
$$

The ground states of the charge- $s$ subspace are given by

$$
|s\rangle=\left\{\begin{array}{ll}
\psi_{s-1} \cdots \psi_{0}|0\rangle & \text { for } s>0, \\
\psi_{s}^{*} \cdots \psi_{-1}^{*}|0\rangle & \text { for } s<0,
\end{array} \quad\langle s|= \begin{cases}\langle 0| \psi_{0}^{*} \cdots \psi_{s-1}^{*} & \text { for } s>0 \\
\langle 0| \psi_{-1} \cdots \psi_{s} & \text { for } s<0\end{cases}\right.
$$

$H_{n}(n \in \mathbf{Z})$ denote the Fourier modes

$$
H_{n}=\sum_{j=-\infty}^{\infty}: \psi_{j} \psi_{j+n}^{*}: \quad \text { (normal ordering) }
$$

of the $U(1)$ current

$$
J(z)=: \psi(z) \psi^{*}(z):=\sum_{n=-\infty}^{\infty} H_{n} z^{-n-1} .
$$

They obey the commutation relations

$$
\left[H_{m}, H_{n}\right]=m \delta_{m+n, 0}
$$

of a Heisenberg algebra.

Solutions of the bilinear equations are now given by

$$
\tau(s, r, \boldsymbol{t}, \overline{\boldsymbol{t}})=\left\langle s+r\left|e^{H(\boldsymbol{t})} g e^{-\bar{H}(\overline{\boldsymbol{t}})}\right| s-r\right\rangle,
$$

where $H(\boldsymbol{t})$ and $\bar{H}(\overline{\boldsymbol{t}})$ are the linear combinations

$$
H(\boldsymbol{t})=\sum_{n=1}^{\infty} t_{n} H_{n}, \quad \bar{H}(\overline{\boldsymbol{t}})=\sum_{n=1}^{\infty} \bar{t}_{n} H_{-n}
$$


of $H_{n}$ 's, and $g$ is an operator of the form

$$
g=\exp \left(\sum_{j, k} a_{j k}: \psi_{j} \psi_{k}^{*}:+\sum_{j, k} b_{j k}: \psi_{j} \psi_{k}:+\sum_{j, k} c_{j k}: \psi_{j}^{*} \psi_{k}^{*}:\right) .
$$

Note that this operator, unlike $H(\boldsymbol{t})$ and $\bar{H}(\overline{\boldsymbol{t}})$, does not preserve charges, hence the foregoing expectation value can take nonzero values for $r \neq 0$.

Let us mention that Willox's original definition $[17,18]$ of the tau function is slightly different from (2.6). His definition reads

$$
\tilde{\tau}(s, r, \boldsymbol{t}, \overline{\boldsymbol{t}})=\left\langle s+r\left|e^{H(t)} e^{\bar{H}(\overline{\boldsymbol{t}})} g e^{-\bar{H}(\overline{\boldsymbol{t}})}\right| s-r\right\rangle .
$$

This is certainly different from our definition; for example, Hirota equations are thereby modified. The difference is, however, minimal, because the two tau functions are connected by the simple relation

$$
\tilde{\tau}(s, r, \boldsymbol{t}, \overline{\boldsymbol{t}})=\exp \left(\sum_{n=1}^{\infty} n t_{n} \bar{t}_{n}\right) \tau(s, r, \boldsymbol{t}, \overline{\boldsymbol{t}}),
$$

so that one can transfer from one definition to the other freely.

The bilinear equation (2.1) is a consequence of the identity

$$
\begin{aligned}
& \oint \frac{d z}{2 \pi i}\left(\psi(z) g \otimes \psi^{*}(z) g+\psi^{*}(z) g \otimes \psi(z) g\right) \\
& \quad=\oint \frac{d z}{2 \pi i}\left(g \psi(z) \otimes g \psi^{*}(z)+g \psi^{*}(z) \otimes g \psi(z)\right)
\end{aligned}
$$

satisfied by the operator $g$. This identity implies the equation

$$
\begin{aligned}
\oint \frac{d z}{2 \pi i}\left\langle s^{\prime}+r^{\prime}+1\left|e^{H^{\prime}} \psi(z) g e^{-\bar{H}^{\prime}}\right| s^{\prime}-r^{\prime}\right\rangle\left\langle s+r-1\left|e^{H} \psi^{*}(z) g e^{-\bar{H}}\right| s-r\right\rangle \\
\quad+\oint \frac{d z}{2 \pi i}\left\langle s^{\prime}+r^{\prime}+1\left|e^{H^{\prime}} \psi^{*}(z) g e^{-\bar{H}^{\prime}}\right| s^{\prime}-r^{\prime}\right\rangle\left\langle s+r-1\left|e^{H} \psi(z) g e^{-\bar{H}}\right| s-r\right\rangle \\
=\oint \frac{d z}{2 \pi i}\left\langle s^{\prime}+r^{\prime}+1\left|e^{H^{\prime}} g \psi(z) e^{-\bar{H}^{\prime}}\right| s^{\prime}-r^{\prime}\right\rangle\left\langle s+r-1\left|e^{H} g \psi^{*}(z) e^{-\bar{H}}\right| s-r\right\rangle \\
\quad+\oint \frac{d z}{2 \pi i}\left\langle s^{\prime}+r^{\prime}+1\left|e^{H^{\prime}} g \psi^{*}(z) e^{-\bar{H}^{\prime}}\right| s^{\prime}-r^{\prime}\right\rangle\left\langle s+r-1\left|e^{H} g \psi(z) e^{-\bar{H}}\right| s^{\prime}-r^{\prime}\right\rangle,
\end{aligned}
$$

where the abbreviated notations

$$
H=H(\boldsymbol{t}), \quad H^{\prime}=H\left(\boldsymbol{t}^{\prime}\right), \quad \bar{H}=\bar{H}(\overline{\boldsymbol{t}}), \quad \bar{H}^{\prime}=\bar{H}\left(\overline{\boldsymbol{t}}^{\prime}\right)
$$

are used. This equation implies the bilinear equation (2.1) by the bosonization formulae

$$
\begin{aligned}
& \langle s| e^{H(\boldsymbol{t})} \psi(z)=z^{s-1} e^{\xi(\boldsymbol{t}, z)}\langle s-1| e^{H\left(\boldsymbol{t}-\left[z^{-1}\right]\right)}, \\
& \langle s| e^{H(\boldsymbol{t})} \psi^{*}(z)=z^{-s-1} e^{-\xi(\boldsymbol{t}, z)}\langle s+1| e^{H\left(\boldsymbol{t}+\left[z^{-1}\right]\right)}
\end{aligned}
$$

and their duals

$$
\begin{aligned}
& \psi(z) e^{-\bar{H}(\overline{\boldsymbol{t}})}|s\rangle=e^{-\bar{H}(\overline{\boldsymbol{t}}-[z])}|s+1\rangle z^{s} e^{\xi\left(\overline{\boldsymbol{t}}, z^{-1}\right)}, \\
& \psi^{*}(z) e^{-\bar{H}(\overline{\boldsymbol{t}})}|s\rangle=e^{-\bar{H}(\overline{\boldsymbol{t}}+[z])}|s-1\rangle z^{-s} e^{-\xi\left(\overline{\boldsymbol{t}}, z^{-1}\right)} .
\end{aligned}
$$




\subsection{Relation to DKP hierarchy}

The Pfaff-Toda hierarchy contains an infinite number of copies of the DKP hierarchy as subsystems.

Such a subsystem shows up by restricting the variables in (2.1) as

$$
\overline{\boldsymbol{t}}^{\prime}=\overline{\boldsymbol{t}}, \quad s=l+r, \quad s^{\prime}=l+r^{\prime},
$$

where $l$ is a constant. (2.1) then reduces to the equation

$$
\begin{aligned}
\oint \frac{d z}{2 \pi i} z^{2 r^{\prime}-2 r} e^{\xi\left(\boldsymbol{t}^{\prime}-\boldsymbol{t}, z\right)} \tau\left(l+r^{\prime}, r^{\prime}, \boldsymbol{t}^{\prime}-\left[z^{-1}\right], \overline{\boldsymbol{t}}\right) \tau\left(l+r, r, \boldsymbol{t}+\left[z^{-1}\right], \overline{\boldsymbol{t}}\right) \\
+\oint \frac{d z}{2 \pi i} z^{2 r-2 r^{\prime}-4} e^{\xi\left(\boldsymbol{t}-\boldsymbol{t}^{\prime}, z\right)} \tau\left(l+r^{\prime}+1, r^{\prime}+1, \boldsymbol{t}^{\prime}+\left[z^{-1}\right], \overline{\boldsymbol{t}}\right) \\
\quad \times \tau\left(l+r-1, r-1, \boldsymbol{t}-\left[z^{-1}\right], \overline{\boldsymbol{t}}\right)=0,
\end{aligned}
$$

which is substantially the bilinear equation characterizing tau functions of the DKP hierarchy. Thus

$$
\tau(l+r, r, \boldsymbol{t}, \overline{\boldsymbol{t}})=\left\langle l+2 r\left|e^{H(\boldsymbol{t})} g e^{-\bar{H}(\overline{\boldsymbol{t}})}\right| l\right\rangle
$$

turns out to be a tau function of the DKP hierarchy with respect to $t$.

One can derive another family of subsystems by restricting the variables as

$$
s=l+1-r, \quad s^{\prime}=l-1-r, \quad \boldsymbol{t}^{\prime}=\boldsymbol{t},
$$

where $l$ is a constant. (2.1) thereby reduces to the equation

$$
\begin{aligned}
0= & \oint \frac{d z}{2 \pi i} z^{-2 r^{\prime}+2 r-2} e^{\xi\left(\overline{\boldsymbol{t}}^{\prime}-\overline{\boldsymbol{t}}, z^{-1}\right)} \tau\left(l-r^{\prime}, r^{\prime}, \boldsymbol{t}, \overline{\boldsymbol{t}}^{\prime}-[z]\right) \tau(l-r, r, \boldsymbol{t}, \overline{\boldsymbol{t}}+[z]) \\
& +\oint \frac{d z}{2 \pi i} z^{-2 r+2 r^{\prime}+2} e^{\xi\left(\overline{\boldsymbol{t}}-\overline{\boldsymbol{t}}^{\prime}, z^{-1}\right)} \tau\left(l-1-r^{\prime}, r^{\prime}+1, \boldsymbol{t}, \overline{\boldsymbol{t}}^{\prime}+[z]\right) \tau(l+1-r, r-1, \boldsymbol{t}, \overline{\boldsymbol{t}}-[z]) .
\end{aligned}
$$

This is again equivalent to the bilinear equation for the DKP hierarchy. Thus

$$
\tau(l-r, r, \boldsymbol{t}, \overline{\boldsymbol{t}})=\left\langle l\left|e^{H(\boldsymbol{t})} g e^{-\bar{H}(\overline{\boldsymbol{t}})}\right| l-2 r\right\rangle
$$

is a tau function of the DKP hierarchy with respect to $\overline{\boldsymbol{t}}$.

\section{Auxiliary linear problem}

\subsection{Wave functions and dressing operators}

To formulate an auxiliary linear problem, we now introduce the wave functions

$$
\begin{aligned}
& \Psi_{1}(s, r, \boldsymbol{t}, \overline{\boldsymbol{t}}, z)=z^{s+r} e^{\xi(\boldsymbol{t}, z)} \frac{\tau\left(s, r, \boldsymbol{t}-\left[z^{-1}\right], \overline{\boldsymbol{t}}\right)}{\tau(s, r, \boldsymbol{t}, \overline{\boldsymbol{t}})}, \\
& \Psi_{2}(s, r, \boldsymbol{t}, \overline{\boldsymbol{t}}, z)=z^{s+r-2} e^{\xi(\boldsymbol{t}, z)} \frac{\tau\left(s-1, r-1, \boldsymbol{t}-\left[z^{-1}\right], \overline{\boldsymbol{t}}\right)}{\tau(s, r, \boldsymbol{t}, \overline{\boldsymbol{t}})}, \\
& \Psi_{1}^{*}(s, r, \boldsymbol{t}, \overline{\boldsymbol{t}}, z)=z^{-s-r-2} e^{-\xi(\boldsymbol{t}, z)} \frac{\tau\left(s+1, r+1, \boldsymbol{t}+\left[z^{-1}\right], \overline{\boldsymbol{t}}\right)}{\tau(s, r, \boldsymbol{t}, \overline{\boldsymbol{t}})}, \\
& \Psi_{2}^{*}(s, r, \boldsymbol{t}, \overline{\boldsymbol{t}}, z)=z^{-s-r} e^{-\xi(\boldsymbol{t}, z)} \frac{\tau\left(s, r, \boldsymbol{t}+\left[z^{-1}\right], \overline{\boldsymbol{t}}\right)}{\tau(s, r, \boldsymbol{t}, \overline{\boldsymbol{t}})}
\end{aligned}
$$


and their duals

$$
\begin{aligned}
& \bar{\Psi}_{1}(s, r, \boldsymbol{t}, \overline{\boldsymbol{t}}, z)=z^{s-r} e^{\xi\left(\overline{\boldsymbol{t}}, z^{-1}\right)} \frac{\tau(s+1, r, \boldsymbol{t}, \overline{\boldsymbol{t}}-[z])}{\tau(s, r, \boldsymbol{t}, \overline{\boldsymbol{t}})}, \\
& \bar{\Psi}_{2}(s, r, \boldsymbol{t}, \overline{\boldsymbol{t}}, z)=z^{s-r} e^{\xi\left(\overline{\boldsymbol{t}}, z^{-1}\right)} \frac{\tau(s, r-1, \boldsymbol{t}, \overline{\boldsymbol{t}}-[z])}{\tau(s, r, \boldsymbol{t}, \overline{\boldsymbol{t}})}, \\
& \bar{\Psi}_{1}^{*}(s, r, \boldsymbol{t}, \overline{\boldsymbol{t}}, z)=z^{-s+r} e^{-\xi\left(\overline{\boldsymbol{t}}, z^{-1}\right)} \frac{\tau(s, r+1, \boldsymbol{t}, \overline{\boldsymbol{t}}+[z])}{\tau(s, r, \boldsymbol{t}, \overline{\boldsymbol{t}})}, \\
& \bar{\Psi}_{2}^{*}(s, r, \boldsymbol{t}, \overline{\boldsymbol{t}}, z)=z^{-s+r} e^{-\xi\left(\overline{\boldsymbol{t}}, z^{-1}\right)} \frac{\tau(s-1, r, \boldsymbol{t}, \overline{\boldsymbol{t}}+[z])}{\tau(s, r, \boldsymbol{t}, \overline{\boldsymbol{t}})} .
\end{aligned}
$$

These wave functions are divided to two groups with respect to the aforementioned two copies of the DKP hierarchy. When the discrete variables $(s, r)$ are restricted on the line $s=l+r$, the first four (3.1) may be thought of as wave functions of the DKP hierarchy with tau function (2.7). Similarly, when $(s, r)$ sit on the line $s=l-r$, the second four (3.2) are to be identified with wave functions of the DKP hierarchy with tau function (2.8). If the tau function is given by the fermionic formula (2.6), these wave functions, too, can be written in a fermionic form as

$$
\begin{aligned}
& \Psi_{1}(s, r, \boldsymbol{t}, \overline{\boldsymbol{t}}, z)=\frac{\left\langle s+r+1\left|e^{H} \psi(z) g e^{-\bar{H}}\right| s-r\right\rangle}{\left\langle s+r\left|e^{H} g e^{-\bar{H}}\right| s-r\right\rangle}, \\
& \Psi_{2}(s, r, \boldsymbol{t}, \overline{\boldsymbol{t}}, z)=\frac{\left\langle s+r-1\left|e^{H} \psi(z) g e^{-\bar{H}}\right| s-r\right\rangle}{\left\langle s+r\left|e^{H} g e^{-\bar{H}}\right| s-r\right\rangle}, \\
& \Psi_{1}^{*}(s, r, \boldsymbol{t}, \overline{\boldsymbol{t}}, z)=\frac{\left\langle s+r+1\left|e^{H} \psi^{*}(z) g e^{-\bar{H}}\right| s-r\right\rangle}{\left\langle s+r\left|e^{H} g e^{-\bar{H}}\right| s-r\right\rangle}, \\
& \Psi_{2}^{*}(s, r, \boldsymbol{t}, \overline{\boldsymbol{t}}, z)=\frac{\left\langle s+r-1\left|e^{H} \psi^{*}(z) g e^{-\bar{H}}\right| s-r\right\rangle}{\left\langle s+r\left|e^{H} g e^{-\bar{H}}\right| s-r\right\rangle},
\end{aligned}
$$

and

$$
\begin{aligned}
\bar{\Psi}_{1}(s, r, \boldsymbol{t}, \overline{\boldsymbol{t}}, z) & =\frac{\left\langle s+r+1\left|e^{H} g \psi(z) e^{-\bar{H}}\right| s-r\right\rangle}{\left\langle s+r\left|e^{H} g e^{-\bar{H}}\right| s-r\right\rangle}, \\
\bar{\Psi}_{2}(s, r, \boldsymbol{t}, \overline{\boldsymbol{t}}, z) & =\frac{\left\langle s+r-1\left|e^{H} g \psi(z) e^{-\bar{H}}\right| s-r\right\rangle}{\left\langle s+r\left|e^{H} g e^{-\bar{H}}\right| s-r\right\rangle}, \\
\bar{\Psi}_{1}^{*}(s, r, \boldsymbol{t}, \overline{\boldsymbol{t}}, z) & =\frac{\left\langle s+r+1\left|e^{H} g \psi^{*}(z) e^{-\bar{H}}\right| s-r\right\rangle}{\left\langle s+r\left|e^{H} g e^{-\bar{H}}\right| s-r\right\rangle}, \\
\bar{\Psi}_{2}^{*}(s, r, \boldsymbol{t}, \overline{\boldsymbol{t}}, z) & =\frac{\left\langle s+r-1\left|e^{H} g \psi^{*}(z) e^{-\bar{H}}\right| s-r\right\rangle}{\left\langle s+r\left|e^{H} g e^{-\bar{H}}\right| s-r\right\rangle} .
\end{aligned}
$$

As a consequence of the bilinear equation (2.1), these wave functions satisfy a system of bilinear equations. Those equations can be cast into a matrix form as

$$
\begin{aligned}
& \oint \frac{d z}{2 \pi i} \Psi_{2 \times 2}\left(s^{\prime}, r^{\prime}, \boldsymbol{t}^{\prime}, \overline{\boldsymbol{t}}^{\prime}, z\right){ }^{\mathrm{t}} \Psi_{2 \times 2}^{*}(s, r, \boldsymbol{t}, \overline{\boldsymbol{t}}, z) \\
& =\oint \frac{d z}{2 \pi i} \bar{\Psi}_{2 \times 2}\left(s^{\prime}, r^{\prime}, \boldsymbol{t}^{\prime}, \overline{\boldsymbol{t}}^{\prime}, z\right){ }^{\mathrm{t}} \bar{\Psi}_{2 \times 2}^{*}(s, r, \boldsymbol{t}, \overline{\boldsymbol{t}}, z),
\end{aligned}
$$

where

$$
\Psi_{2 \times 2}(s, r, \boldsymbol{t}, \overline{\boldsymbol{t}}, z)=\left(\begin{array}{ll}
\Psi_{1}(s, r, \boldsymbol{t}, \overline{\boldsymbol{t}}, z) & \Psi_{1}^{*}(s, r, \boldsymbol{t}, \overline{\boldsymbol{t}}, z) \\
\Psi_{2}(s, r, \boldsymbol{t}, \overline{\boldsymbol{t}}, z) & \Psi_{2}^{*}(s, r, \boldsymbol{t}, \overline{\boldsymbol{t}}, z)
\end{array}\right),
$$




$$
\begin{aligned}
\Psi_{2 \times 2}^{*}(s, r, \boldsymbol{t}, \overline{\boldsymbol{t}}, z) & =\left(\begin{array}{ll}
\Psi_{1}^{*}(s, r, \boldsymbol{t}, \overline{\boldsymbol{t}}, z) & \Psi_{1}(s, r, \boldsymbol{t}, \overline{\boldsymbol{t}}, z) \\
\Psi_{2}^{*}(s, r, \boldsymbol{t}, \overline{\boldsymbol{t}}, z) & \Psi_{2}(s, r, \boldsymbol{t}, \overline{\boldsymbol{t}}, z)
\end{array}\right), \\
\bar{\Psi}_{2 \times 2}(s, r, \boldsymbol{t}, \overline{\boldsymbol{t}}, z) & =\left(\begin{array}{ll}
\bar{\Psi}_{1}(s, r, \boldsymbol{t}, \overline{\boldsymbol{t}}, z) & \bar{\Psi}_{1}^{*}(s, r, \boldsymbol{t}, \overline{\boldsymbol{t}}, z) \\
\bar{\Psi}_{2}(s, r, \boldsymbol{t}, \overline{\boldsymbol{t}}, z) & \bar{\Psi}_{2}^{*}(s, r, \boldsymbol{t}, \overline{\boldsymbol{t}}, z)
\end{array}\right), \\
\bar{\Psi}_{2 \times 2}^{*}(s, r, \boldsymbol{t}, \overline{\boldsymbol{t}}, z) & =\left(\begin{array}{ll}
\bar{\Psi}_{1}^{*}(s, r, \boldsymbol{t}, \overline{\boldsymbol{t}}, z) & \bar{\Psi}_{1}(s, r, \boldsymbol{t}, \overline{\boldsymbol{t}}, z) \\
\bar{\Psi}_{2}^{*}(s, r, \boldsymbol{t}, \overline{\boldsymbol{t}}, z) & \bar{\Psi}_{2}(s, r, \boldsymbol{t}, \overline{\boldsymbol{t}}, z)
\end{array}\right) .
\end{aligned}
$$

Let us now introduce the dressing operators

$$
\begin{array}{ll}
W_{1}=1+\sum_{n=1}^{\infty} w_{1 n} e^{-n \partial_{s}}, & V_{1}=\sum_{n=0}^{\infty} v_{1 n} e^{(n+2) \partial_{s}}, \\
W_{2}=\sum_{n=0}^{\infty} w_{2 n} e^{-(n+2) \partial_{s}}, & V_{2}=1+\sum_{n=1}^{\infty} v_{2 n} e^{n \partial_{s}}, \\
\bar{W}_{1}=\sum_{n=0}^{\infty} \bar{w}_{1 n} e^{n \partial_{s}}, & \bar{V}_{1}=\sum_{n=0}^{\infty} \bar{v}_{1 n} e^{-n \partial_{s}}, \\
\bar{W}_{2}=\sum_{n=0}^{\infty} \bar{w}_{2 n} e^{n \partial_{s}}, & \bar{V}_{2}=\sum_{n=0}^{\infty} \bar{v}_{2 n} e^{-n \partial_{s}},
\end{array}
$$

where $w_{1 n}$, etc., are the coefficients of Laurent expansion of the tau-quotient in the wave functions (3.1) and (3.2), namely,

$$
\begin{array}{ll}
\frac{\tau\left(s, r, \boldsymbol{t}-\left[z^{-1}\right], \overline{\boldsymbol{t}}\right)}{\tau(s, r, \boldsymbol{t}, \overline{\boldsymbol{t}})}=1+\sum_{n=1}^{\infty} w_{1 n} z^{-n}, & \frac{\tau\left(s+1, r+1, \boldsymbol{t}+\left[z^{-1}\right], \overline{\boldsymbol{t}}\right)}{\tau(s, r, \boldsymbol{t}, \overline{\boldsymbol{t}})}=\sum_{n=0}^{\infty} v_{1 n} z^{-n}, \\
\frac{\tau\left(s-1, r-1, \boldsymbol{t}-\left[z^{-1}\right], \overline{\boldsymbol{t}}\right)}{\tau(s, r, \boldsymbol{t}, \overline{\boldsymbol{t}})}=\sum_{n=0}^{\infty} w_{2 n} z^{-n}, & \frac{\tau\left(s, r, \boldsymbol{t}+\left[z^{-1}\right], \overline{\boldsymbol{t}}\right)}{\tau(s, r, \boldsymbol{t}, \overline{\boldsymbol{t}})}=1+\sum_{n=1}^{\infty} v_{2 n} z^{-n},
\end{array}
$$

and

$$
\begin{array}{ll}
\frac{\tau(s+1, r, \boldsymbol{t}, \overline{\boldsymbol{t}}-[z])}{\tau(s, r, \boldsymbol{t}, \overline{\boldsymbol{t}})}=\sum_{n=0}^{\infty} \bar{w}_{1 n} z^{n}, & \frac{\tau(s, r+1, \boldsymbol{t}, \overline{\boldsymbol{t}}+[z])}{\tau(s, r, \boldsymbol{t}, \overline{\boldsymbol{t}})}=\sum_{n=0}^{\infty} \bar{v}_{1 n} z^{n}, \\
\frac{\tau(s, r-1, \boldsymbol{t}, \overline{\boldsymbol{t}}-[z])}{\tau(s, r, \boldsymbol{t}, \overline{\boldsymbol{t}})}=\sum_{n=0}^{\infty} \bar{w}_{2 n} z^{n}, & \frac{\tau(s-1, r, \boldsymbol{t}, \overline{\boldsymbol{t}}+[z])}{\tau(s, r, \boldsymbol{t}, \overline{\boldsymbol{t}})}=\sum_{n=0}^{\infty} \bar{v}_{2 n} z^{n} .
\end{array}
$$

The wave function can be thereby expressed as

$$
\begin{array}{ll}
\Psi_{\alpha}(s, r, \boldsymbol{t}, \overline{\boldsymbol{t}}, z)=W_{\alpha} z^{s+r} e^{\xi(\boldsymbol{t}, z)}, & \bar{\Psi}_{\alpha}(s, r, \boldsymbol{t}, \overline{\boldsymbol{t}}, z)=\bar{W}_{\alpha} z^{s-r} e^{\xi\left(\overline{\boldsymbol{t}}, z^{-1}\right)} \\
\Psi_{\alpha}^{*}(s, r, \boldsymbol{t}, \overline{\boldsymbol{t}}, z)=V_{\alpha} z^{-s-r} e^{-\xi(\boldsymbol{t}, z)}, & \bar{\Psi}_{\alpha}^{*}(s, r, \boldsymbol{t}, \overline{\boldsymbol{t}}, z)=\bar{V}_{\alpha} z^{-s+r} e^{-\xi\left(\overline{\boldsymbol{t}}, z^{-1}\right)} .
\end{array}
$$

\subsection{Algebraic relations among dressing operators}

A technical clue of the following consideration is a formula that connects wave functions and dressing operators. This formula is an analogue of the formula for the case where the dressing operators are pseudo-differential operators [30,31, 32]. Let us introduce a few notations. For a pair of difference operators of the form

$$
P=\sum_{n=-\infty}^{\infty} p_{n}(s) e^{n \partial_{s}}, \quad Q=\sum_{n=-\infty}^{\infty} q_{n}(s) e^{n \partial_{s}}
$$


let $\Psi(s, z)$ and $\Phi(s, z)$ denote the wave functions

$$
\Psi(s, z)=P z^{s}=\sum_{n=-\infty}^{\infty} p_{n}(s) z^{n+s}, \quad \Phi(s, z)=Q z^{-s}=\sum_{n=-\infty}^{\infty} q_{n}(s) z^{-n-s} .
$$

Moreover, let $P^{*}$ denote the formal adjoint

$$
P^{*}=\sum_{n=-\infty}^{\infty} e^{-n \partial_{s}} p_{n}(s),
$$

and $(P)_{s^{\prime} s}$ the "matrix elements"

$$
(P)_{s^{\prime} s}=p_{s-s^{\prime}}\left(s^{\prime}\right) \text {. }
$$

With these notations, the formula reads

$$
\oint \frac{d z}{2 \pi i} \Psi\left(s^{\prime}, z\right) \Phi(s, z)=\left(P e^{\partial_{s}} Q^{*}\right)_{s^{\prime} s}=\left(Q e^{-\partial_{s}} P^{*}\right)_{s s^{\prime}} .
$$

One can derive this formula by straightforward calculations, which are rather simpler than the case of pseudo-differential operators [30, 31, 32].

To illustrate the usage of this formula, we now derive a set of algebraic relations satisfied by the dressing operators from the bilinear equation (3.3) specialized to $\boldsymbol{t}^{\prime}=\boldsymbol{t}$ and $\overline{\boldsymbol{t}}^{\prime}=\overline{\boldsymbol{t}}$. Since these relations contain dressing operators for two different values of $r$, let us indicate the $(s, r)$ dependence explicitly as $W(s, r)$, etc.

Theorem 1. Specialization of the bilinear equation (3.3) to $\boldsymbol{t}^{\prime}=\boldsymbol{t}$ and $\overline{\boldsymbol{t}}^{\prime}=\overline{\boldsymbol{t}}$ is equivalent to the algebraic relations

$$
\begin{aligned}
& W_{\alpha}\left(s, r^{\prime}\right) e^{\left(r^{\prime}-r+1\right) \partial_{s}} V_{\beta}(s, r)^{*}+V_{\alpha}\left(s, r^{\prime}\right) e^{\left(r^{\prime}-r-1\right) \partial_{s}} W_{\beta}(s, r)^{*} \\
& \quad=\bar{W}_{\alpha}\left(s, r^{\prime}\right) e^{\left(r-r^{\prime}+1\right) \partial_{s}} \bar{V}_{\beta}(s, r)^{*}+\bar{V}_{\alpha}\left(s, r^{\prime}\right) e^{\left(r-r^{\prime}-1\right) \partial_{s}} \bar{W}_{\beta}(s, r)^{*}
\end{aligned}
$$

for $\alpha, \beta=1,2$.

Proof. The $(1,1)$ component of the specialized bilinear equation reads

$$
\begin{aligned}
& \oint \frac{d z}{2 \pi i} \Psi_{1}\left(s^{\prime}, r^{\prime}, z\right) \Psi_{1}^{*}(s, r, z)+\oint \frac{d z}{2 \pi i} \Psi_{1}^{*}\left(s^{\prime}, r^{\prime}, z\right) \Psi_{1}(s, r, z) \\
& =\oint \frac{d z}{2 \pi i} \bar{\Psi}_{1}\left(s^{\prime}, r^{\prime}, z\right) \bar{\Psi}_{1}^{*}(s, r, z)+\oint \frac{d z}{2 \pi i} \bar{\Psi}_{1}^{*}\left(s^{\prime}, r^{\prime}, z\right) \bar{\Psi}_{1}(s, r, z) .
\end{aligned}
$$

By the key formula (3.4), each term of this equation can be expressed as

$$
\begin{aligned}
\oint \frac{d z}{2 \pi i} \Psi_{1}\left(s^{\prime}, r^{\prime}, z\right) \Psi_{1}^{*}(s, r, z) & =\left(W_{1}\left(s, r^{\prime}\right) e^{\left(r^{\prime}-r+1\right) \partial_{s}} V_{1}(s, r)\right)_{s^{\prime} s}, \\
\oint \frac{d z}{2 \pi i} \Psi_{1}^{*}\left(s^{\prime}, r^{\prime}, z\right) \Psi_{1}(s, r, z) & =\left(W_{1}(s, r) e^{\left(r-r^{\prime}+1\right) \partial_{s}} V_{1}\left(s, r^{\prime}\right)\right)_{s s^{\prime}} \\
& =\left(V_{1}\left(s, r^{\prime}\right) e^{\left(r^{\prime}-r-1\right) \partial_{s}} W_{1}(s, r)\right)_{s^{\prime} s} \\
\oint \frac{d z}{2 \pi i} \bar{\Psi}_{1}\left(s^{\prime}, r^{\prime}, z\right) \bar{\Psi}_{1}^{*}(s, r, z) & =\left(\bar{W}_{1}\left(s, r^{\prime}\right) e^{\left(r-r^{\prime}+1\right) \partial_{s}} \bar{V}_{1}(s, r)\right)_{s^{\prime} s} \\
\oint \frac{d z}{2 \pi i} \bar{\Psi}_{1}^{*}\left(s^{\prime}, r^{\prime}, z\right) \bar{\Psi}_{1}(s, r, z) & =\left(\bar{W}_{1}(s, r) e^{\left(r^{\prime}-r+1\right) \partial_{s}} \bar{V}_{1}\left(s, r^{\prime}\right)\right)_{s s^{\prime}} \\
& =\left(\bar{V}_{1}\left(s, r^{\prime}\right) e^{\left(r-r^{\prime}-1\right) \partial_{s}} \bar{W}_{1}(s, r)\right)_{s^{\prime} s} .
\end{aligned}
$$

Thus we find that the $(1,1)$ component of the specialized bilinear equation is equivalent to $(3.5)$ for $\alpha=\beta=1$. The other components can be treated in the same way. 
In particular, letting $r^{\prime}=r$ in (3.5), we obtain a set of algebraic relations satisfied by $W, V$, $\bar{W}, \bar{V}$. We can rewrite these relations in the following matrix form, which turns out to be useful later on. Note that the formal adjoint of a matrix of operators is defined to be the transposed matrix of the formal adjoints of matrix elements as

$$
\left(\begin{array}{cc}
A & B \\
C & D
\end{array}\right)^{*}=\left(\begin{array}{cc}
A^{*} & C^{*} \\
B^{*} & D^{*}
\end{array}\right)
$$

Corollary 1. The dressing operators satisfy the algebraic relation

$$
\left(\begin{array}{ll}
W_{1} & \bar{V}_{1} \\
W_{2} & \bar{V}_{2}
\end{array}\right)^{*}=\left(\begin{array}{cc}
0 & e^{\partial_{s}} \\
-e^{-\partial_{s}} & 0
\end{array}\right)\left(\begin{array}{ll}
\bar{W}_{1} & V_{1} \\
\bar{W}_{2} & V_{2}
\end{array}\right)^{-1}\left(\begin{array}{cc}
0 & -e^{\partial_{s}} \\
e^{-\partial_{s}} & 0
\end{array}\right)
$$

or, equivalently,

$$
\left(\begin{array}{ll}
\bar{W}_{1} & V_{1} \\
\bar{W}_{2} & V_{2}
\end{array}\right)^{*}=\left(\begin{array}{cc}
0 & e^{\partial_{s}} \\
-e^{-\partial_{s}} & 0
\end{array}\right)\left(\begin{array}{ll}
W_{1} & \bar{V}_{1} \\
W_{2} & \bar{V}_{2}
\end{array}\right)^{-1}\left(\begin{array}{cc}
0 & -e^{\partial_{s}} \\
e^{-\partial_{s}} & 0
\end{array}\right) .
$$

Proof. Let us examine (3.5) in the case where $r^{\prime}=r$. The $(1,1)$ component reads

$$
W_{1} e^{\partial_{s}} V_{1}^{*}+V_{1} e^{-\partial_{s}} W_{1}^{*}=\bar{W}_{1} e^{\partial_{s}} \bar{V}_{1}^{*}+\bar{V}_{1} e^{-\partial_{s}} \bar{W}_{1}^{*} .
$$

Among the four terms in this relation, $W_{1} e^{\partial_{s}} V_{1}^{*}$ and $\bar{V}_{1} e^{-\partial_{s}} \bar{W}_{1}^{*}$ are linear combinations of $e^{-\partial_{s}}, e^{-2 \partial_{s}}, \ldots$, and $V_{1} e^{-\partial_{s}} W_{1}^{*}$ and $\bar{W}_{1} e^{\partial_{s}} \bar{V}_{1}^{*}$ are linear combinations of $e^{\partial_{s}}, e^{2 \partial_{s}}, \ldots$ Therefore this relation splits into the two relations

$$
V_{1} e^{-\partial_{s}} W_{1}^{*}=\bar{W}_{1} e^{\partial_{s}} \bar{V}_{1}^{*}, \quad W_{1} e^{\partial_{s}} V_{1}^{*}=\bar{V}_{1} e^{-\partial_{s}} \bar{W}_{1}^{*},
$$

which are actually equivalent. In the same way, we can derive the relations

$$
V_{2} e^{-\partial_{s}} W_{2}^{*}=\bar{W}_{2} e^{\partial_{s}} \bar{V}_{2}^{*}, \quad W_{2} e^{\partial_{s}} V_{2}^{*}=\bar{V}_{2} e^{-\partial_{s}} \bar{W}_{2}^{*}
$$

from the $(2,2)$ component of $(3.5)$. Let us now consider the $(1,2)$ component, which we rewrite as

$$
W_{1} e^{\partial_{s}} V_{2}^{*}-\bar{V}_{1} e^{-\partial_{s}} \bar{W}_{2}^{*}=\bar{W}_{1} e^{\partial_{s}} \bar{V}_{2}^{*}-V_{1} e^{-\partial_{s}} W_{2}^{*}
$$

The left hand side is a sum of $e^{\partial_{s}}$ and a linear combination of $1, e^{-\partial_{s}}, \ldots$, and the right hand side is a sum of $e^{\partial_{s}}$ and a linear combination of $e^{2 \partial_{s}}, e^{3 \partial_{s}}, \ldots$. Therefore both hand sides should be equal to $e^{\partial_{s}}$, namely,

$$
W_{1} e^{\partial_{s}} V_{2}^{*}-\bar{V}_{1} e^{-\partial_{s}} \bar{W}_{2}^{*}=\bar{W}_{1} e^{\partial_{s}} \bar{V}_{2}^{*}-V_{1} e^{-\partial_{s}} W_{2}^{*}=e^{\partial_{s}} .
$$

By the same reasoning, we can derive the relations

$$
W_{2} e^{\partial_{s}} V_{1}^{*}-\bar{V}_{2} e^{-\partial_{s}} \bar{W}_{1}^{*}=\bar{W}_{2} e^{\partial_{s}} \bar{V}_{1}^{*}-V_{2} e^{-\partial_{s}} W_{1}^{*}=-e^{-\partial_{s}}
$$

from the $(2,1)$ component of $(3.5)$. These relations can be cast into a matrix form as (3.6) and (3.7).

(3.6) and (3.7) may be thought of as constraints preserved under time evolutions with respect to $\boldsymbol{t}$ and $\overline{\boldsymbol{t}}$. Actually, the discrete variable $r$, too, has to be interpreted as a time variable. Letting $r^{\prime}=r+1$ in (3.5), we can see how the dressing operators evolve in $r$. 
Corollary 2. The dressing operators with $r$ shifted by one are related to the unshifted dressing operators as

$$
\begin{aligned}
& \left(\begin{array}{ll}
W_{1}(s, r+1) & \bar{V}_{1}(s, r+1) \\
W_{2}(s, r+1) & \bar{V}_{2}(s, r+1)
\end{array}\right)\left(\begin{array}{cc}
e^{\partial_{s}} & 0 \\
0 & e^{-\partial_{s}}
\end{array}\right)=\left(\begin{array}{cc}
A & B \\
C & 0
\end{array}\right)\left(\begin{array}{ll}
W_{1} & \bar{V}_{1} \\
W_{2} & \bar{V}_{2}
\end{array}\right), \\
& \left(\begin{array}{ll}
\bar{W}_{1}(s, r+1) & V_{1}(s, r+1) \\
\bar{W}_{2}(s, r+1) & V_{2}(s, r+1)
\end{array}\right)\left(\begin{array}{cc}
e^{-\partial_{s}} & 0 \\
0 & e^{\partial_{s}}
\end{array}\right)=\left(\begin{array}{cc}
A & B \\
C & 0
\end{array}\right)\left(\begin{array}{ll}
\bar{W}_{1} & V_{1} \\
\bar{W}_{2} & V_{2}
\end{array}\right),
\end{aligned}
$$

where

$$
\begin{aligned}
& A=e^{\partial_{s}}+\left(\log \frac{\tau(s+1, r)}{\tau(s, r+1)}\right)_{t_{1}}+\frac{\tau(s+1, r+1) \tau(s-1, r)}{\tau(s, r+1) \tau(s, r)} e^{-\partial_{s}}, \\
& B=-\frac{\tau(s+1, r+1)}{\tau(s, r)} e^{\partial_{s}}, \quad C=\frac{\tau(s-1, r)}{\tau(s, r+1)} e^{-\partial_{s}} .
\end{aligned}
$$

Proof. When $r^{\prime}=r+1$, (3.5) reads

$$
W_{\alpha}(s, r+1) e^{2 \partial_{s}} V_{\beta}^{*}+V_{\alpha}(s, r+1) W_{\beta}^{*}=\bar{W}_{\alpha}(s, r+1) \bar{V}_{\beta}^{*}+\bar{V}_{\alpha}(s, r+1) e^{-2 \partial_{s}} \bar{W}_{\beta}^{*} .
$$

These equations can be cast into a matrix form as

$$
\begin{gathered}
\left(\begin{array}{ll}
W_{1}(s, r+1) & \bar{V}_{1}(s, r+1) \\
W_{2}(s, r+1) & \bar{V}_{2}(s, r+1)
\end{array}\right)\left(\begin{array}{cc}
0 & e^{2 \partial_{s}} \\
-e^{-2 \partial_{s}} & 0
\end{array}\right)\left(\begin{array}{cc}
\bar{W}_{1}^{*} & \bar{W}_{2}^{*} \\
V_{1}^{*} & V_{2}^{*}
\end{array}\right) \\
\quad=\left(\begin{array}{ll}
\bar{W}_{1}(s, r+1) & V_{1}(s, r+1) \\
\bar{W}_{2}(s, r+1) & V_{2}(s, r+1)
\end{array}\right)\left(\begin{array}{cc}
0 & 1 \\
-1 & 0
\end{array}\right)\left(\begin{array}{cc}
W_{1}^{*} & W_{2}^{*} \\
\bar{V}_{1}^{*} & \bar{V}_{2}^{*}
\end{array}\right) .
\end{gathered}
$$

Noting that

$$
\left(\begin{array}{cc}
\bar{W}_{1}^{*} & \bar{W}_{2}^{*} \\
V_{1}^{*} & V_{2}^{*}
\end{array}\right)=\left(\begin{array}{ll}
\bar{W}_{1} & V_{1} \\
\bar{W}_{2} & V_{2}
\end{array}\right)^{*}, \quad\left(\begin{array}{cc}
W_{1}^{*} & W_{2}^{*} \\
\bar{V}_{1}^{*} & \bar{V}_{2}^{*}
\end{array}\right)=\left(\begin{array}{cc}
W_{1} & \bar{V}_{1} \\
W_{2} & \bar{V}_{2}
\end{array}\right)^{*}
$$

we can use (3.6) and (3.7) to rewrite this equation as

$$
\begin{aligned}
& \left(\begin{array}{ll}
W_{1}(s, r+1) & \bar{V}_{1}(s, r+1) \\
W_{2}(s, r+1) & \bar{V}_{2}(s, r+1)
\end{array}\right)\left(\begin{array}{cc}
e^{\partial_{s}} & 0 \\
0 & e^{-\partial_{s}}
\end{array}\right)\left(\begin{array}{cc}
W_{1} & \bar{V}_{1} \\
W_{2} & \bar{V}_{2}
\end{array}\right)^{-1} \\
& \quad=\left(\begin{array}{ll}
\bar{W}_{1}(s, r+1) & V_{1}(s, r+1) \\
\bar{W}_{2}(s, r+1) & V_{2}(s, r+1)
\end{array}\right)\left(\begin{array}{cc}
e^{-\partial_{s}} & 0 \\
0 & e^{\partial_{s}}
\end{array}\right)\left(\begin{array}{ll}
\bar{W}_{1} & V_{1} \\
\bar{W}_{2} & V_{2}
\end{array}\right)^{-1} .
\end{aligned}
$$

By the definition of the dressing operators, the left hand side of this equation is a matrix of operators of the form

$$
\left(\begin{array}{cc}
e^{\partial_{s}}+w_{11}(s, r+1)-w_{11}(s+1, r)+\cdots & -\frac{\bar{v}_{10}(s+1, r)}{\bar{v}_{20}(s+1, r)} e^{\partial_{s}}+\cdots \\
\bullet e^{-\partial_{s}}+\bullet e^{-2 \partial_{s}}+\cdots & \bullet e^{-\partial_{s}}+\bullet e^{-2 \partial_{s}}+\cdots
\end{array}\right)
$$

( denotes a function), and the right hand side take such a form as

$$
\left(\begin{array}{cc}
\frac{\bar{w}_{10}(s, r+1)}{\bar{w}_{10}(s-1, r)} e^{-\partial_{s}}+\cdots & \bullet e^{\partial_{s}}+\bullet e^{2 \partial_{s}}+\cdots \\
\frac{\bar{w}_{20}(s, r+1)}{\bar{w}_{10}(s-1, r)} e^{-\partial_{s}}+\cdots & \bullet e^{\partial_{s}}+\bullet e^{2 \partial_{s}}+\cdots
\end{array}\right) .
$$

Consequently,

$$
\left(\begin{array}{ll}
W_{1}(s, r+1) & \bar{V}_{1}(s, r+1) \\
W_{2}(s, r+1) & V_{2}(s, r+1)
\end{array}\right)\left(\begin{array}{cc}
e^{\partial_{s}} & 0 \\
0 & e^{-\partial_{s}}
\end{array}\right)\left(\begin{array}{ll}
W_{1} & \bar{V}_{1} \\
W_{2} & \bar{V}_{2}
\end{array}\right)^{-1}=\left(\begin{array}{cc}
A & B \\
C & 0
\end{array}\right)
$$


and

$$
\left(\begin{array}{cc}
\bar{W}_{1}(s, r+1) & V_{1}(s, r+1) \\
\bar{W}_{2}(s, r+1) & V_{2}(s, r+1)
\end{array}\right)\left(\begin{array}{cc}
e^{-\partial_{s}} & 0 \\
0 & e^{\partial_{s}}
\end{array}\right)\left(\begin{array}{ll}
\bar{W}_{1} & V_{1} \\
\bar{W}_{2} & V_{2}
\end{array}\right)^{-1}=\left(\begin{array}{cc}
A & B \\
C & 0
\end{array}\right),
$$

where

$$
\begin{aligned}
& A=e^{\partial_{s}}+w_{11}(s, r+1)-w_{11}(s+1, r)+\frac{\bar{w}_{10}(s, r+1)}{\bar{w}_{10}(s-1, r)} e^{-\partial_{s}}, \\
& B=-\frac{\bar{v}_{10}(s+1, r)}{\bar{v}_{20}(s+1, r)} e^{\partial_{s}}, \quad C=w_{20}(s, r+1) e^{-\partial_{s}}=\frac{\bar{w}_{20}(s, r+1)}{\bar{w}_{10}(s-1, r)} e^{-\partial_{s}} .
\end{aligned}
$$

Rewriting this result in terms of the tau functions, we obtain the formulae (3.9) of $A, B, C$.

\subsection{Evolution equations of dressing operators}

The dressing operators turn out to satisfy a set of evolution equations with respect to the continuous time variables $\boldsymbol{t}$ and $\overline{\boldsymbol{t}}$ as well. To present the result, let us introduce the notations

$$
\begin{aligned}
(P)_{\geq 0} & =\sum_{n \geq 0} p_{n} e^{n \partial_{s}}, & (P)_{>0} & =\sum_{n>0} p_{n} e^{n \partial_{s}}, \\
(P)_{\leq 0} & =\sum_{n \leq 0} p_{n} e^{n \partial_{s}}, & (P)_{<0} & =\sum_{n<0} p_{n} e^{n \partial_{s}}
\end{aligned}
$$

of truncated operators for difference operators of the form

$$
P=\sum_{n=-\infty}^{\infty} p_{n} e^{n \partial_{s}}
$$

Theorem 2. The dressing operators satisfy the equations

$$
\begin{aligned}
& \left(\begin{array}{cc}
W_{1, t_{n}}+W_{1} e^{n \partial_{s}} & \bar{V}_{1, t_{n}} \\
W_{2, t_{n}}+W_{2} e^{n \partial_{s}} & \bar{V}_{2, t_{n}}
\end{array}\right)=\left(\begin{array}{cc}
A_{n} & B_{n} \\
C_{n} & D_{n}
\end{array}\right)\left(\begin{array}{ll}
W_{1} & \bar{V}_{1} \\
W_{2} & \bar{V}_{2}
\end{array}\right), \\
& \left(\begin{array}{cc}
\bar{W}_{1, t_{n}} & V_{1, t_{n}}-V_{1} e^{-n \partial_{s}} \\
\bar{W}_{2, t_{n}} & V_{2, t_{n}}-V_{2} e^{-n \partial_{s}}
\end{array}\right)=\left(\begin{array}{cc}
A_{n} & B_{n} \\
C_{n} & D_{n}
\end{array}\right)\left(\begin{array}{cc}
\bar{W}_{1} & V_{1} \\
\bar{W}_{2} & V_{2}
\end{array}\right),
\end{aligned}
$$

where the subscript $t_{n}$ in $W_{1, t_{n}}$, etc., stands for differentiating the coefficients of the difference operators by $t_{n}$,

$$
W_{\alpha, t_{n}}=\frac{\partial W_{\alpha}}{\partial t_{n}}, \quad V_{\alpha, t_{n}}=\frac{\partial V_{\alpha}}{\partial t_{n}}, \quad \bar{W}_{\alpha, t_{n}}=\frac{\partial \bar{W}_{\alpha}}{\partial t_{n}}, \quad \bar{V}_{\alpha, t_{n}}=\frac{\partial \bar{V}_{\alpha}}{\partial t_{n}},
$$

and $A_{n}, B_{n}, C_{n}$ and $D_{n}$ are defined as

$$
\begin{aligned}
& A_{n}=\left(W_{1} e^{(n+1) \partial_{s}} V_{2}^{*} e^{-\partial_{s}}\right)_{\geq 0}+\left(V_{1} e^{-(n+1) \partial_{s}} W_{2}^{*} e^{-\partial_{s}}\right)_{<0}, \\
& B_{n}=-\left(W_{1} e^{(n+1) \partial_{s}} V_{1}^{*} e^{\partial_{s}}\right)_{>0}-\left(V_{1} e^{-(n+1) \partial_{s}} W_{1}^{*} e^{\partial_{s}}\right)_{\leq 0}, \\
& C_{n}=\left(W_{2} e^{(n+1) \partial_{s}} V_{2}^{*} e^{-\partial_{s}}\right)_{\geq 0}+\left(V_{2} e^{-(n+1) \partial_{s}} W_{2}^{*} e^{-\partial_{s}}\right)_{<0}, \\
& D_{n}=-\left(W_{2} e^{-(n+1) \partial_{s}} V_{1}^{*} e^{\partial_{s}}\right)_{>0}-\left(V_{2} e^{-(n+1) \partial_{s}} W_{1}^{*} e^{\partial_{s}}\right)_{\leq 0} .
\end{aligned}
$$

Proof. Differentiate the bilinear equation (3.3) by $t_{n}^{\prime}$ and specialize the variables to $\boldsymbol{t}^{\prime}=\boldsymbol{t}$, $\overline{\boldsymbol{t}}^{\prime}=\overline{\boldsymbol{t}}, s^{\prime}=s$ and $r^{\prime}=r$. This leads to the equation

$$
\oint \frac{d z}{2 \pi i} \partial_{t_{n}} \Psi_{2 \times 2}(s, r, z) \cdot{ }^{\mathrm{t}} \Psi_{2 \times 2}^{*}(s, r, z)=\oint \frac{d z}{2 \pi i} \partial_{t_{n}} \bar{\Psi}_{2 \times 2}^{*}(s, r, z) \cdot{ }^{\mathrm{t}} \bar{\Psi}_{2 \times 2}^{*}(s, r, z) .
$$


The $t_{n}$-derivatives of the wave functions in this equation can be expressed as

$$
\begin{aligned}
& \partial_{t_{n}} \Psi_{\alpha}(s, r, z)=\left(W_{\alpha, t_{n}}+W_{\alpha} e^{n \partial_{s}}\right) z^{s+r} e^{\xi(\boldsymbol{t}, z)}, \\
& \partial_{t_{n}} \Psi_{\alpha}^{*}(s, r, z)=\left(V_{\alpha, t_{n}}-V_{\alpha} e^{-n \partial_{s}}\right) z^{-s-r} e^{-\xi(\boldsymbol{t}, z)}, \\
& \partial_{t_{n}} \bar{\Psi}_{\alpha}(s, r, z)=\bar{W}_{\alpha, t_{n}} z^{s-r} e^{\xi\left(\overline{\boldsymbol{t}}, z^{-1}\right)}, \\
& \partial_{t_{n}} \bar{\Psi}_{\alpha}^{*}(s, r, z)=\bar{V}_{\alpha, t_{n}} e^{-s+r} e^{-\xi\left(\overline{\boldsymbol{t}}, z^{-1}\right)} .
\end{aligned}
$$

We now use the key formula (3.4) to convert the last equation to equations for the dressing operators. Those equations can be cast into a matrix form as

$$
\begin{gathered}
\left(\begin{array}{cc}
W_{1, t_{n}}+W_{1} e^{n \partial_{s}} & \bar{V}_{1, t_{n}} \\
W_{2, t_{n}}+W_{2} e^{n \partial_{s}} & \bar{V}_{1, t_{n}}
\end{array}\right)\left(\begin{array}{cc}
0 & -e^{\partial_{s}} \\
e^{-\partial_{s}} & 0
\end{array}\right)\left(\begin{array}{cc}
\bar{W}_{1}^{*} & \bar{W}_{2}^{*} \\
V_{1}^{*} & V_{2}^{*}
\end{array}\right) \\
=\left(\begin{array}{ll}
\bar{W}_{1, t_{n}} & V_{1, t_{n}}-V_{1} e^{-n \partial_{s}} \\
\bar{W}_{2, t_{n}} & V_{2, t_{n}}-V_{2} e^{-n \partial_{s}}
\end{array}\right)\left(\begin{array}{cc}
0 & -e^{\partial_{s}} \\
e^{-\partial_{s}} & 0
\end{array}\right)\left(\begin{array}{cc}
W_{1}^{*} & W_{2}^{*} \\
\bar{V}_{1}^{*} & \bar{V}_{2}^{*}
\end{array}\right) .
\end{gathered}
$$

By (3.6) and (3.7), this equation can be rewritten as

$$
\begin{aligned}
& \left(\begin{array}{cc}
W_{1, t_{n}}+W_{1} e^{n \partial_{s}} & \bar{V}_{1, t_{n}} \\
W_{2, t_{n}}+W_{2} e^{n \partial_{s}} & \bar{V}_{1, t_{n}}
\end{array}\right)\left(\begin{array}{cc}
W_{1} & \bar{V}_{1} \\
W_{2} & \bar{V}_{2}
\end{array}\right)^{-1} \\
& =\left(\begin{array}{ll}
\bar{W}_{1, t_{n}} & V_{1, t_{n}}-V_{1} e^{-n \partial_{s}} \\
\bar{W}_{2, t_{n}} & V_{2, t_{n}}-V_{2} e^{-n \partial_{s}}
\end{array}\right)\left(\begin{array}{ll}
\bar{W}_{1} & V_{1} \\
\bar{W}_{2} & V_{2}
\end{array}\right)^{-1} .
\end{aligned}
$$

Let $A_{n}, B_{n}, C_{n}, D_{n}$ denote the matrix elements of both hand sides of this equation. To complete the proof, we have to show that these difference operators do have the form stated in the theorem. To this end, let us compare the two different expressions

$$
\begin{aligned}
\left(\begin{array}{cc}
A_{n} & B_{n} \\
C_{n} & D_{n}
\end{array}\right) & =\left(\begin{array}{ll}
W_{1, t_{n}}+W_{1} e^{n \partial_{s}} & \bar{V}_{1, t_{n}} \\
W_{2, t_{n}}+W_{2} e^{n \partial_{s}} & \bar{V}_{2, t_{n}}
\end{array}\right)\left(\begin{array}{cc}
W_{1} & \bar{V}_{1} \\
W_{2} & \bar{V}_{2}
\end{array}\right)^{-1} \\
& =\left(\begin{array}{ll}
W_{1, t_{n}}+W_{1} e^{n \partial_{s}} & \bar{V}_{1, t_{n}} \\
W_{2, t_{n}}+W_{2} e^{n \partial_{s}} & \bar{V}_{2, t_{n}}
\end{array}\right)\left(\begin{array}{cc}
e^{\partial_{s}} V_{2}^{*} e^{-\partial_{s}} & -e^{\partial_{s}} V_{1}^{*} e^{\partial_{s}} \\
-e^{-\partial_{s}} \bar{W}_{2}^{*} e^{-\partial_{s}} & e^{-\partial_{s}} \bar{W}_{1}^{*} e^{\partial_{s}}
\end{array}\right)
\end{aligned}
$$

and

$$
\begin{aligned}
\left(\begin{array}{cc}
A_{n} & B_{n} \\
C_{n} & D_{n}
\end{array}\right) & =\left(\begin{array}{ll}
\bar{W}_{1, t_{n}} & V_{1, t_{n}}-V_{1} e^{-n \partial_{s}} \\
\bar{W}_{2, t_{n}} & V_{2, t_{n}}-V_{2} e^{-n \partial_{s}}
\end{array}\right)\left(\begin{array}{cc}
\bar{W}_{1} & V_{1} \\
\bar{W}_{2} & V_{2}
\end{array}\right)^{-1} \\
& =\left(\begin{array}{ll}
\bar{W}_{1, t_{n}} & V_{1, t_{n}}-V_{1} e^{-n \partial_{s}} \\
\bar{W}_{2, t_{n}} & V_{2, t_{n}}-V_{2} e^{-n \partial_{s}}
\end{array}\right)\left(\begin{array}{cc}
e^{\partial_{s}} \bar{V}_{2}^{*} e^{-\partial_{s}} & -e^{\partial_{s}} \bar{V}_{1}^{*} e^{\partial_{s}} \\
-e^{-\partial_{s}} W_{2}^{*} e^{-\partial_{s}} & e^{-\partial_{s}} W_{1}^{*} e^{\partial_{s}}
\end{array}\right)
\end{aligned}
$$

of the matrix of these operators that can be derived from the foregoing construction and the algebraic relations (3.6) and (3.7). As regards $A_{n}$, this implies that

$$
\begin{aligned}
A_{n} & =\left(W_{1, t_{n}}+W_{1} e^{n \partial_{s}}\right) e^{\partial_{s}} V_{2}^{*} e^{-\partial_{s}}-\bar{V}_{1, t_{n}} e^{-\partial_{s}} \bar{W}_{2}^{*} e^{-\partial_{s}} \\
& =\bar{W}_{1, t_{n}} e^{\partial_{s}} \bar{V}_{2}^{*} e^{-\partial_{s}}-\left(V_{1, t_{n}}-V_{1} e^{-n \partial_{s}}\right) e^{-\partial_{s}} W_{2}^{*} e^{-\partial_{s}} .
\end{aligned}
$$

From the ()$\geq 0$ part of the first line, we have the identity

$$
\left(A_{n}\right)_{\geq 0}=\left(W_{1} e^{(n+1) \partial_{s}} V_{2}^{*} e^{-\partial_{s}}\right)_{\geq 0},
$$

and from the ()$_{<0}$ part of the second line, similarly,

$$
\left(A_{n}\right)_{<0}=\left(V_{1} e^{-(n+1) \partial_{s}} W_{2}^{*} e^{-\partial_{s}}\right)_{<0} .
$$

Thus $A_{n}$ turns out to be given by the sum of these operators as

$$
A_{n}=\left(W_{1} e^{(n+1) \partial_{s}} V_{2}^{*} e^{-\partial_{s}}\right)_{\geq 0}+\left(V_{1} e^{-(n+1) \partial_{s}} W_{2}^{*} e^{-\partial_{s}}\right)_{<0} .
$$

The other operators $B_{n}, C_{n}, D_{n}$, too, can be identified in a fully parallel manner. 
In much the same way, the following evolution equations in $\overline{\boldsymbol{t}}$ can be derived.

Theorem 3. The dressing operators satisfy the equations

$$
\begin{aligned}
& \left(\begin{array}{cc}
W_{1, \bar{t}_{n}} & \bar{V}_{1, \bar{t}_{n}}-\bar{V}_{n} e^{n \partial_{s}} \\
W_{2, \bar{t}_{n}} & \bar{V}_{2, \bar{t}_{n}}-\bar{V}_{2} e^{n \partial_{s}}
\end{array}\right)=\left(\begin{array}{cc}
\bar{A}_{n} & \bar{B}_{n} \\
\bar{C}_{n} & \bar{D}_{n}
\end{array}\right)\left(\begin{array}{cc}
W_{1} & \bar{V}_{1} \\
W_{2} & \bar{V}_{2}
\end{array}\right), \\
& \left(\begin{array}{cc}
\bar{W}_{1, \bar{t}_{n}}+\bar{W}_{1} e^{-n \partial_{s}} & V_{1, \bar{t}_{n}} \\
\bar{W}_{2, \bar{t}_{n}}+\bar{W}_{2} e^{-n \partial_{s}} & V_{2, \bar{t}_{n}}
\end{array}\right)=\left(\begin{array}{cc}
\bar{A}_{n} & \bar{B}_{n} \\
\bar{C}_{n} & \bar{D}_{n}
\end{array}\right)\left(\begin{array}{ll}
\bar{W}_{1} & V_{1} \\
\bar{W}_{2} & V_{2}
\end{array}\right),
\end{aligned}
$$

where

$$
\begin{aligned}
& \bar{A}_{n}=\left(\bar{W}_{1} e^{-(n-1) \partial_{s}} \bar{V}_{2}^{*} e^{-\partial_{s}}\right)_{<0}+\left(\bar{V}_{1} e^{(n-1) \partial_{s}} \bar{W}_{2}^{*} e^{-\partial_{s}}\right)_{\geq 0}, \\
& \bar{B}_{n}=-\left(\bar{W}_{1} e^{-(n-1) \partial_{s}} \bar{V}_{1}^{*} e^{\partial_{s}}\right)_{\leq 0}-\left(\bar{V}_{1} e^{(n-1) \partial_{s}} \bar{W}_{1}^{*} e^{\partial_{s}}\right)_{>0}, \\
& \bar{C}_{n}=\left(\bar{W}_{2} e^{-(n-1) \partial_{s}} \bar{V}_{2}^{*} e^{-\partial_{s}}\right)_{<0}+\left(\bar{V}_{2} e^{(n-1) \partial_{s}} \bar{W}_{2}^{*} e^{-\partial_{s}}\right)_{\geq 0}, \\
& \bar{D}_{n}=-\left(\bar{W}_{2} e^{-(n-1) \partial_{s}} \bar{V}_{1}^{*} e^{\partial_{s}}\right)_{\leq 0}-\left(\bar{V}_{2} e^{(n-1) \partial_{s}} \bar{W}_{1}^{*} e^{\partial_{s}}\right)_{>0} .
\end{aligned}
$$

\subsection{Auxiliary linear equations}

The evolution equations (3.10), (3.11) and (3.8) for the dressing operators can be readily cast into auxiliary linear equations for the wave functions.

Corollary 3. The wave functions satisfy the following linear equations:

$$
\begin{aligned}
e^{\partial_{r}}\left(\begin{array}{llll}
\Psi_{1} & \Psi_{1}^{*} & \bar{\Psi}_{1} & \bar{\Psi}_{1}^{*} \\
\Psi_{2} & \Psi_{2}^{*} & \bar{\Psi}_{2} & \bar{\Psi}_{2}^{*}
\end{array}\right) & =\left(\begin{array}{cc}
A & B \\
C & 0
\end{array}\right)\left(\begin{array}{llll}
\Psi_{1} & \Psi_{1}^{*} & \bar{\Psi}_{1} & \bar{\Psi}_{1}^{*} \\
\Psi_{2} & \Psi_{2}^{*} & \bar{\Psi}_{2} & \bar{\Psi}_{2}^{*}
\end{array}\right), \\
\partial_{t_{n}}\left(\begin{array}{llll}
\Psi_{1} & \Psi_{1}^{*} & \bar{\Psi}_{1} & \bar{\Psi}_{1}^{*} \\
\Psi_{2} & \Psi_{2}^{*} & \bar{\Psi}_{2} & \bar{\Psi}_{2}^{*}
\end{array}\right) & =\left(\begin{array}{ccc}
A_{n} & B_{n} \\
C_{n} & D_{n}
\end{array}\right)\left(\begin{array}{llll}
\Psi_{1} & \Psi_{1}^{*} & \bar{\Psi}_{1} & \bar{\Psi}_{1}^{*} \\
\Psi_{2} & \Psi_{2}^{*} & \bar{\Psi}_{2} & \bar{\Psi}_{2}^{*}
\end{array}\right), \\
\partial_{\bar{t}_{n}}\left(\begin{array}{lllll}
\Psi_{1} & \Psi_{1}^{*} & \bar{\Psi}_{1} & \bar{\Psi}_{1}^{*} \\
\Psi_{2} & \Psi_{2}^{*} & \bar{\Psi}_{2} & \bar{\Psi}_{2}^{*}
\end{array}\right) & =\left(\begin{array}{llll}
\bar{A}_{n} & \bar{B}_{n} \\
\bar{C}_{n} & \bar{D}_{n}
\end{array}\right)\left(\begin{array}{llll}
\Psi_{1} & \Psi_{1}^{*} & \bar{\Psi}_{1} & \bar{\Psi}_{1}^{*} \\
\Psi_{2} & \Psi_{2}^{*} & \bar{\Psi}_{2} & \bar{\Psi}_{2}^{*}
\end{array}\right) .
\end{aligned}
$$

Note that each of (3.13), (3.14) and (3.12) is a collective expression of four sets of linear equations, namely,

$$
\begin{aligned}
& \left(\begin{array}{l}
e^{\partial_{r}} \Phi_{1} \\
e^{\partial_{r}} \Phi_{2}
\end{array}\right)=\left(\begin{array}{cc}
A & B \\
C & 0
\end{array}\right)\left(\begin{array}{l}
\Phi_{1} \\
\Phi_{2}
\end{array}\right), \quad\left(\begin{array}{c}
\partial_{t_{n}} \Phi_{1} \\
\partial_{t_{n}} \Phi_{2}
\end{array}\right)=\left(\begin{array}{cc}
A_{n} & B_{n} \\
C_{n} & D_{n}
\end{array}\right)\left(\begin{array}{l}
\Phi_{1} \\
\Phi_{2}
\end{array}\right), \\
& \left(\begin{array}{c}
\partial_{\bar{t}_{n}} \Phi_{1} \\
\partial_{\bar{t}_{n}} \Phi_{2}
\end{array}\right)=\left(\begin{array}{ll}
\bar{A}_{n} & \bar{B}_{n} \\
\bar{C}_{n} & \bar{D}_{n}
\end{array}\right)\left(\begin{array}{l}
\Phi_{1} \\
\Phi_{2}
\end{array}\right)
\end{aligned}
$$

for the four pairs $\Phi_{\alpha}=\Psi_{\alpha}, \Psi_{\alpha}^{*}, \bar{\Psi}_{\alpha}, \bar{\Psi}_{\alpha}^{*}(\alpha=1,2)$ of wave functions. The lowest $(n=1)$ equations of (3.13) and (3.14) agree with Willox's result $[17,18]$ :

$$
\begin{aligned}
& \left(\begin{array}{c}
\partial_{t_{1}} \Phi_{1}(s, r, \mu) \\
\partial_{t_{1}} \Phi_{2}(s, r, \mu)
\end{array}\right) \\
& \quad=\left(\begin{array}{cc}
e^{\partial_{s}}+\left(\log \frac{\tau(s+1, r)}{\tau(s, r)}\right)_{t_{1}} & -\frac{\tau(s+1, r+1)}{\tau(s, r)} e^{\partial_{s}} \\
\frac{\tau(s-1, r-1)}{\tau(s, r)} e^{-\partial_{s}} & -e^{-\partial_{s}}+\left(\log \frac{\tau(s-1, r)}{\tau(s, r)}\right)_{t_{1}}
\end{array}\right)\left(\begin{array}{l}
\Phi_{1}(s, r, \mu) \\
\Phi_{2}(s, r, \mu)
\end{array}\right), \\
& \left(\begin{array}{cc}
\partial_{\bar{t}_{1}} \Phi_{1}(s, r, \mu) \\
\partial_{\bar{t}_{1}} \Phi_{2}(s, r, \mu)
\end{array}\right) \\
& \quad=\left(\begin{array}{cc}
\frac{\tau(s+1, r) \tau(s-1, r)}{\tau(s, r)^{2}} e^{-\partial_{s}} & -\frac{\tau(s+1, r) \tau(s, r+1)}{\tau(s, r)^{2}} e^{\partial_{s}} \\
\frac{\tau(s-1, r) \tau(s, r-1)}{\tau(s, r)^{2}} e^{-\partial_{s}} & -\frac{\tau(s+1, r) \tau(s-1, r)}{\tau(s, r)^{2}} e^{\partial_{s}}
\end{array}\right)\left(\begin{array}{l}
\Phi_{1}(s, r, \mu) \\
\Phi_{2}(s, r, \mu)
\end{array}\right) .
\end{aligned}
$$


Thus we have obtained auxiliary linear equations for the Pfaff-Toda hierarchy. Apart from the fact that difference operators play a central role, this auxiliary linear problem resembles that of the DKP hierarchy (see Appendix). This is a manifestation of the common Lie algebraic structure $[2,3]$ that underlies these hierarchies.

Let us specify an algebraic structure in the building blocks of the auxiliary linear problem. Let $U, \bar{U}, P_{n}, \bar{P}_{n}$ and $J$ denote the matrix operators

$$
\begin{array}{ll}
U=\left(\begin{array}{ll}
W_{1} & \bar{V}_{1} \\
W_{2} & \bar{V}_{2}
\end{array}\right), \quad \bar{U}=\left(\begin{array}{ll}
\bar{W}_{1} & V_{1} \\
\bar{W}_{2} & V_{2}
\end{array}\right), \quad P_{n}=\left(\begin{array}{cc}
A_{n} & B_{n} \\
C_{n} & D_{n}
\end{array}\right), \\
\bar{P}_{n}=\left(\begin{array}{cc}
\bar{A}_{n} & \bar{B}_{n} \\
\bar{C}_{n} & \bar{D}_{n}
\end{array}\right), & J=\left(\begin{array}{cc}
0 & e^{\partial_{s}} \\
-e^{-\partial_{s}} & 0
\end{array}\right), \quad J^{-1}=-J .
\end{array}
$$

With these notations, (3.6) can be rewritten as

$$
U^{*}=J \bar{U}^{-1} J^{-1}, \quad \bar{U}^{*}=J U^{-1} J^{-1} .
$$

This exhibits a Lie group structure (now realized in terms of difference operators). Moreover, (3.10) and (3.11) imply that $P_{n}$ and $\bar{P}_{n}$ can be expressed as

$$
\begin{aligned}
& P_{n}=U_{t_{n}} U^{-1}+U\left(\begin{array}{cc}
e^{n \partial_{s}} & 0 \\
0 & 0
\end{array}\right) U^{-1}=\bar{U}_{t_{n}} \bar{U}^{-1}+\bar{U}\left(\begin{array}{cc}
0 & 0 \\
0 & -e^{-n \partial_{s}}
\end{array}\right) \bar{U}^{-1}, \\
& \bar{P}_{n}=U_{\bar{t}_{n}} U^{-1}+U\left(\begin{array}{cc}
0 & 0 \\
0 & -e^{n \partial_{s}}
\end{array}\right) U^{-1}=\bar{U}_{\bar{t}_{n}} \bar{U}^{-1}+\bar{U}\left(\begin{array}{cc}
e^{-n \partial_{s}} & 0 \\
0 & 0
\end{array}\right) \bar{U}^{-1} .
\end{aligned}
$$

We can confirm by straightforward calculations that $P_{n}$ and $\bar{P}_{n}$ satisfy the algebraic relations

$$
P_{n}^{*}=-J P_{n} J^{-1}, \quad \bar{P}_{n}^{*}=-J \bar{P}_{n} J^{-1},
$$

which are obviously a Lie algebraic version of the foregoing constraints for $U$ and $\bar{U}$. In components, these relations read

$$
A_{n}^{*}=-e^{\partial_{s}} D_{n} e^{-\partial_{s}}, \quad B_{n}^{*}=e^{-\partial_{s}} B_{n} e^{-\partial_{s}}, \quad C_{n}^{*}=e^{\partial_{s}} C_{n} e^{\partial_{s}}, \quad D_{n}^{*}=-e^{-\partial_{s}} A_{n} e^{\partial_{s}},
$$

and

$$
\bar{A}_{n}^{*}=-e^{\partial_{s}} \bar{D}_{n} e^{-\partial_{s}}, \quad \bar{B}_{n}^{*}=e^{-\partial_{s}} \bar{B}_{n} e^{-\partial_{s}}, \quad \bar{C}_{n}^{*}=e^{\partial_{s}} \bar{C}_{n} e^{\partial_{s}}, \quad \bar{D}_{n}^{*}=-e^{-\partial_{s}} \bar{A}_{n} e^{\partial_{s}} .
$$

These relations are parallel to algebraic relations in the case of the DKP hierarchy (see Appendix).

\section{Difference Fay identities}

\subsection{How to derive difference Fay identities}

We now derive six Fay-like identities with parameters $\lambda$ and $\mu$ from the bilinear equation (2.2) by specializing the free variables therein as follows:

1a) $\boldsymbol{t}^{\prime}=\boldsymbol{t}+\left[\lambda^{-1}\right]+\left[\mu^{-1}\right], \overline{\boldsymbol{t}}^{\prime}=\overline{\boldsymbol{t}}, s^{\prime}=s+1, r^{\prime}=r$;

1b) $\boldsymbol{t}^{\prime}=\boldsymbol{t}+\left[\lambda^{-1}\right]+\left[\mu^{-1}\right], \overline{\boldsymbol{t}}^{\prime}=\overline{\boldsymbol{t}}, s^{\prime}=s, r^{\prime}=r+1$;

2a) $\boldsymbol{t}^{\prime}=\boldsymbol{t}, \overline{\boldsymbol{t}}^{\prime}=\overline{\boldsymbol{t}}+[\lambda]+[\mu], s^{\prime}=s-1, r^{\prime}=r$;

2b) $\boldsymbol{t}^{\prime}=\boldsymbol{t}, \overline{\boldsymbol{t}}^{\prime}=\overline{\boldsymbol{t}}+[\lambda]+[\mu], s^{\prime}=s, r^{\prime}=r+1$;

3a) $\boldsymbol{t}^{\prime}=\boldsymbol{t}+\left[\lambda^{-1}\right], \overline{\boldsymbol{t}}^{\prime}=\overline{\boldsymbol{t}}+[\mu], s^{\prime}=s, r^{\prime}=r$;

3b) $\boldsymbol{t}^{\prime}=\boldsymbol{t}+\left[\lambda^{-1}\right], \overline{\boldsymbol{t}}^{\prime}=\overline{\boldsymbol{t}}+[\mu], s^{\prime}=s-1, r^{\prime}=r+1$.

To clarify the meaning of calculations, we now assume that the integrals in (2.2) are contour integrals along simple closed curves $C_{\infty}$ and $C_{0}$ encircling the points $z=\infty$ and $z=0$ respectively. $\lambda$ and $\mu$ are understood to be in a particular position specified below. 
1a) and 1b). $\lambda$ and $\mu$ are assumed to sit on the far side (closer to $z=\infty$ ) of the contour $C_{\infty}$. The exponential factors in the integrand thereby become rational functions as

$$
e^{\xi\left(\boldsymbol{t}^{\prime}-\boldsymbol{t}, z\right)}=\frac{1}{(1-z / \lambda)(1-z / \mu)}, \quad e^{\xi\left(\overline{\boldsymbol{t}}^{\prime}-\overline{\boldsymbol{t}}, z^{-1}\right)}=1 .
$$

Thus the bilinear equation (2.2) reduce to the equations

$$
\begin{aligned}
& \oint_{C_{\infty}} \frac{d z}{2 \pi i} \frac{\lambda \mu}{z(z-\lambda)(z-\mu)} \tau\left(s+1, r, \boldsymbol{t}+\left[\lambda^{-1}\right]+\left[\mu^{-1}\right]-\left[z^{-1}\right], \overline{\boldsymbol{t}}\right) \tau\left(s+1, r+1, \boldsymbol{t}+\left[z^{-1}\right], \overline{\boldsymbol{t}}\right) \\
& \quad+\oint_{C_{\infty}} \frac{d z}{2 \pi i} \frac{(z-\lambda)(z-\mu)}{z^{3} \lambda \mu} \tau\left(s+2, r+1, \boldsymbol{t}+\left[\lambda^{-1}\right]+\left[\mu^{-1}\right]+\left[z^{-1}\right], \overline{\boldsymbol{t}}\right) \tau\left(s, r, \boldsymbol{t}-\left[z^{-1}\right], \overline{\boldsymbol{t}}\right) \\
& =\oint_{C_{0}} \frac{d z}{2 \pi i} z \tau\left(s+2, r, \boldsymbol{t}+\left[\lambda^{-1}\right]+\left[\mu^{-1}\right], \overline{\boldsymbol{t}}-[z]\right) \tau(s, r+1, \boldsymbol{t}, \overline{\boldsymbol{t}}+[z]) \\
& \quad+\oint_{C_{0}} \frac{d z}{2 \pi i} \frac{1}{z} \tau\left(s+1, r+1, \boldsymbol{t}+\left[\lambda^{-1}\right]+\left[\mu^{-1}\right], \overline{\boldsymbol{t}}+[z]\right) \tau(s+1, r, \boldsymbol{t}, \overline{\boldsymbol{t}}-[z])
\end{aligned}
$$

in the case of 1a) and

$$
\begin{aligned}
& \oint_{C_{\infty}} \frac{d z}{2 \pi i} \frac{\lambda \mu}{z(z-\lambda)(z-\mu)} \tau\left(s, r+1, \boldsymbol{t}+\left[\lambda^{-1}\right]+\left[\mu^{-1}\right]-\left[z^{-1}\right], \overline{\boldsymbol{t}}\right) \tau\left(s+1, r+1, \boldsymbol{t}+\left[z^{-1}\right], \overline{\boldsymbol{t}}\right) \\
& \quad+\oint_{C_{\infty}} \frac{d z}{2 \pi i} \frac{(z-\lambda)(z-\mu)}{z^{3} \lambda \mu} \tau\left(s+1, r+2, \boldsymbol{t}+\left[\lambda^{-1}\right]+\left[\mu^{-1}\right]+\left[z^{-1}\right], \overline{\boldsymbol{t}}\right) \tau\left(s, r, \boldsymbol{t}-\left[z^{-1}\right], \overline{\boldsymbol{t}}\right) \\
& =\oint_{C_{0}} \frac{d z}{2 \pi i} \frac{1}{z} \tau\left(s+1, r+1, \boldsymbol{t}+\left[\lambda^{-1}\right]+\left[\mu^{-1}\right], \overline{\boldsymbol{t}}-[z]\right) \tau(s, r+1, \boldsymbol{t}, \overline{\boldsymbol{t}}+[z]) \\
& \quad+\oint_{C_{0}} \frac{d z}{2 \pi i} z \tau\left(s, r+2, \boldsymbol{t}+\left[\lambda^{-1}\right]+\left[\mu^{-1}\right], \overline{\boldsymbol{t}}+[z]\right) \tau(s+1, r, \boldsymbol{t}, \overline{\boldsymbol{t}}-[z])
\end{aligned}
$$

in the case of $1 \mathrm{~b})$. The contour integrals in these equation can be calculated by residue calculus. For example, the first integral on the left hand side is given by the sum of residues of the integrand at $z=\lambda, \mu$; the other contour integrals can be treated in the same way. The outcome are the equations

$$
\begin{aligned}
& -\frac{\mu}{\lambda-\mu} \tau\left(s+1, r, \boldsymbol{t}+\left[\mu^{-1}\right], \overline{\boldsymbol{t}}\right) \tau\left(s+1, r+1, \boldsymbol{t}+\left[\lambda^{-1}\right], \overline{\boldsymbol{t}}\right) \\
& -\frac{\lambda}{\mu-\lambda} \tau\left(s+1, r, \boldsymbol{t}+\left[\lambda^{-1}\right], \overline{\boldsymbol{t}}\right) \tau\left(s+1, r+1, \boldsymbol{t}+\left[\mu^{-1}\right], \overline{\boldsymbol{t}}\right) \\
& +\frac{1}{\lambda \mu} \tau\left(s+2, r+1, \boldsymbol{t}+\left[\lambda^{-1}\right]+\left[\mu^{-1}\right], \overline{\boldsymbol{t}}\right) \tau(s, r, \boldsymbol{t}, \overline{\boldsymbol{t}}) \\
& =\tau\left(s+1, r+1, \boldsymbol{t}+\left[\lambda^{-1}\right]+\left[\mu^{-1}\right], \overline{\boldsymbol{t}}\right) \tau(s+1, r, \boldsymbol{t}, \overline{\boldsymbol{t}}), \\
& -\frac{\mu}{\lambda-\mu} \tau\left(s, r+1, \boldsymbol{t}+\left[\mu^{-1}\right], \overline{\boldsymbol{t}}\right) \tau\left(s+1, r+1, \boldsymbol{t}+\left[\lambda^{-1}\right], \overline{\boldsymbol{t}}\right) \\
& -\frac{\lambda}{\mu-\lambda} \tau\left(s, r+1, \boldsymbol{t}+\left[\lambda^{-1}\right], \overline{\boldsymbol{t}}\right) \tau\left(s+1, r+1, \boldsymbol{t}+\left[\mu^{-1}\right], \overline{\boldsymbol{t}}\right) \\
& +\frac{1}{\lambda \mu} \tau\left(s+1, r+2, \boldsymbol{t}+\left[\lambda^{-1}\right]+\left[\mu^{-1}\right], \overline{\boldsymbol{t}}\right) \tau(s, r, \boldsymbol{t}, \overline{\boldsymbol{t}}) \\
& =\tau\left(s+1, r+1, \boldsymbol{t}+\left[\lambda^{-1}\right]+\left[\mu^{-1}\right], \overline{\boldsymbol{t}}\right) \tau(s, r+1, \boldsymbol{t}, \overline{\boldsymbol{t}}) .
\end{aligned}
$$

2a) and $\mathbf{2 b}$ ). $\quad \lambda$ and $\mu$ are assumed to be inside $C_{0}$ (nearer to $z=0$ ). The bilinear equation (2.2) turn into the equations

$$
\oint_{C_{\infty}} \frac{d z}{2 \pi i} \frac{1}{z^{3}} \tau\left(s-1, r, \boldsymbol{t}-\left[z^{-1}\right], \overline{\boldsymbol{t}}+[\lambda]+[\mu]\right) \tau\left(s+1, r+1, \boldsymbol{t}+\left[z^{-1}\right], \overline{\boldsymbol{t}}\right)
$$




$$
\begin{aligned}
& +\oint_{C_{\infty}} \frac{d z}{2 \pi i} \frac{1}{z} \tau\left(s, r+1, \boldsymbol{t}+\left[z^{-1}\right], \overline{\boldsymbol{t}}+[\lambda]+[\mu]\right) \tau\left(s, r, \boldsymbol{t}-\left[z^{-1}\right], \overline{\boldsymbol{t}}\right) \\
= & \oint_{C_{0}} \frac{d z}{2 \pi i} \frac{z}{(z-\lambda)(z-\mu)} \tau(s, r, \boldsymbol{t}, \overline{\boldsymbol{t}}+[\lambda]+[\mu]-[z]) \tau(s, r+1, \boldsymbol{t}, \overline{\boldsymbol{t}}+[z]) \\
& +\oint_{C_{0}} \frac{d z}{2 \pi i} \frac{(z-\lambda)(z-\mu)}{z} \tau(s-1, r+1, \boldsymbol{t}, \overline{\boldsymbol{t}}+[\lambda]+[\mu]+[z]) \tau(s+1, r, \boldsymbol{t}, \overline{\boldsymbol{t}}-[z])
\end{aligned}
$$

in the case of $2 \mathrm{a}$ ) and

$$
\begin{aligned}
\oint_{C_{\infty}} & \frac{d z}{2 \pi i} \frac{1}{z} \tau\left(s, r+1, \boldsymbol{t}-\left[z^{-1}\right], \overline{\boldsymbol{t}}+[\lambda]+[\mu]\right) \tau\left(s+1, r+1, \boldsymbol{t}+\left[z^{-1}\right], \overline{\boldsymbol{t}}\right) \\
& +\oint_{C_{\infty}} \frac{d z}{2 \pi i} \frac{1}{z^{3}} \tau\left(s+1, r+2, \boldsymbol{t}+\left[z^{-1}\right], \overline{\boldsymbol{t}}+[\lambda]+[\mu]\right) \tau\left(s, r, \boldsymbol{t}-\left[z^{-1}\right], \overline{\boldsymbol{t}}\right) \\
= & \oint_{C_{0}} \frac{d z}{2 \pi i} \frac{z}{(z-\lambda)(z-\mu)} \tau(s+1, r+1, \boldsymbol{t}, \overline{\boldsymbol{t}}+[\lambda]+[\mu]-[z]) \tau(s, r+1, \boldsymbol{t}, \overline{\boldsymbol{t}}+[z]) \\
& +\oint_{C_{0}} \frac{d z}{2 \pi i} \frac{(z-\lambda)(z-\mu)}{z} \tau(s, r+2, \boldsymbol{t}, \overline{\boldsymbol{t}}+[\lambda]+[\mu]+[z]) \tau(s+1, r, \boldsymbol{t}, \overline{\boldsymbol{t}}-[z])
\end{aligned}
$$

in the case of $2 \mathrm{~b})$. By residue calculus, we obtain the equations

$$
\begin{aligned}
\tau(s, r & +1, \boldsymbol{t}, \overline{\boldsymbol{t}}+[\lambda]+[\mu]) \tau(s, r, \boldsymbol{t}, \overline{\boldsymbol{t}}) \\
= & \frac{\lambda}{\lambda-\mu} \tau(s, r, \boldsymbol{t}, \overline{\boldsymbol{t}}+[\mu]) \tau(s, r+1, \boldsymbol{t}, \overline{\boldsymbol{t}}+[\lambda]) \\
& +\frac{\mu}{\mu-\lambda} \tau(s, r, \boldsymbol{t}, \overline{\boldsymbol{t}}+[\lambda]) \tau(s, r+1, \boldsymbol{t}, \overline{\boldsymbol{t}}+[\mu]) \\
& +\lambda \mu \tau(s-1, r+1, \boldsymbol{t}, \overline{\boldsymbol{t}}+[\lambda]+[\mu]) \tau(s+1, r, \boldsymbol{t}, \overline{\boldsymbol{t}}), \\
\tau(s, r & +1, \boldsymbol{t}, \overline{\boldsymbol{t}}+[\lambda]+[\mu]) \tau(s+1, r+1, \boldsymbol{t}, \overline{\boldsymbol{t}}) \\
& =\frac{\lambda}{\lambda-\mu} \tau(s+1, r+1, \boldsymbol{t}, \overline{\boldsymbol{t}}+[\mu]) \tau(s, r+1, \boldsymbol{t}, \overline{\boldsymbol{t}}+[\lambda]) \\
& +\frac{\mu}{\mu-\lambda} \tau(s+1, r+1, \boldsymbol{t}, \overline{\boldsymbol{t}}+[\lambda]) \tau(s, r+1, \boldsymbol{t}, \overline{\boldsymbol{t}}+[\mu]) \\
& +\lambda \mu \tau(s, r+2, \boldsymbol{t}, \overline{\boldsymbol{t}}+[\lambda]+[\mu]) \tau(s+1, r, \boldsymbol{t}, \overline{\boldsymbol{t}}) .
\end{aligned}
$$

3a) and 3b). $\lambda$ and $\mu$ are assumed to be on the far side of $C_{\infty}$ and inside $C_{0}$ respectively. The bilinear equation (2.2) turn into the equations

$$
\begin{aligned}
& \oint_{C_{\infty}} \frac{d z}{2 \pi i} \\
& \quad+\oint_{C_{\infty}} \frac{d z}{z^{2}(z-\lambda)} \tau\left(s, r, \boldsymbol{t}+\left[\lambda^{-1}\right]-\left[z^{-1}\right], \overline{\boldsymbol{t}}+[\mu]\right) \tau\left(s+1, r+1, \boldsymbol{t}+\left[z^{-1}\right], \overline{\boldsymbol{t}}\right) \\
&=\oint_{C_{0}} \frac{d z}{2 \pi i} \frac{z}{z-\mu} \tau\left(s+1, r+1, \boldsymbol{t}+\left[\lambda^{-1}\right]+\left[z^{-1}\right], \overline{\boldsymbol{t}}+[\mu]\right) \tau\left(s, r, \boldsymbol{t}-\left[z^{-1}\right], \overline{\boldsymbol{t}}\right) \\
& \quad+\oint_{C_{0}} \frac{d z}{2 \pi i} \frac{z-\mu}{z} \tau\left(s, r+\left[\lambda^{-1}\right], \overline{\boldsymbol{t}}+[\mu]-[z]\right) \tau(s, r+1, \boldsymbol{t}, \overline{\boldsymbol{t}}+[z])
\end{aligned}
$$

in the case of $3 \mathrm{a}$ ) and

$$
\oint_{C_{\infty}} \frac{d z}{2 \pi i} \frac{-\lambda}{z^{2}(z-\lambda)} \tau\left(s-1, r+1, \boldsymbol{t}+\left[\lambda^{-1}\right]-\left[z^{-1}\right], \overline{\boldsymbol{t}}+[\mu]\right) \tau\left(s+1, r+1, \boldsymbol{t}+\left[z^{-1}\right], \overline{\boldsymbol{t}}\right)
$$




$$
\begin{aligned}
& +\oint_{C_{\infty}} \frac{d z}{2 \pi i} \frac{(z-\lambda)}{-z^{2} \lambda} \tau\left(s, r+2, \boldsymbol{t}+\left[\lambda^{-1}\right]+\left[z^{-1}\right], \overline{\boldsymbol{t}}+[\mu]\right) \tau\left(s, r, \boldsymbol{t}-\left[z^{-1}\right], \overline{\boldsymbol{t}}\right) \\
= & \oint_{C_{0}} \frac{d z}{2 \pi i} \frac{1}{z(z-\mu)} \tau\left(s, r+1, \boldsymbol{t}+\left[\lambda^{-1}\right], \overline{\boldsymbol{t}}+[\mu]-[z]\right) \tau(s, r+1, \boldsymbol{t}, \overline{\boldsymbol{t}}+[z]) \\
& +\oint_{C_{0}} \frac{d z}{2 \pi i} z(z-\mu) \tau\left(s-1, r+2, \boldsymbol{t}+\left[\lambda^{-1}\right], \overline{\boldsymbol{t}}+[\mu]+[z]\right) \tau(s+1, r, \boldsymbol{t}, \overline{\boldsymbol{t}}-[z])
\end{aligned}
$$

in the case of $3 \mathrm{~b})$, and boil down to the equations

$$
\begin{aligned}
\lambda^{-1} \tau & (s, r, \boldsymbol{t}, \overline{\boldsymbol{t}}+[\mu]) \tau\left(s+1, r+1, \boldsymbol{t}+\left[\lambda^{-1}\right], \overline{\boldsymbol{t}}\right) \\
& -\lambda^{-1} \tau\left(s+1, r+1, \boldsymbol{t}+\left[\lambda^{-1}\right], \overline{\boldsymbol{t}}+[\mu]\right) \tau(s, r, \boldsymbol{t}, \overline{\boldsymbol{t}}) \\
& =\mu \tau\left(s+1, r, \boldsymbol{t}+\left[\lambda^{-1}\right], \overline{\boldsymbol{t}}\right) \tau(s, r+1, \boldsymbol{t}, \overline{\boldsymbol{t}}+[\mu]) \\
& -\mu \tau\left(s, r+1, \boldsymbol{t}+\left[\lambda^{-1}\right], \overline{\boldsymbol{t}}+[\mu]\right) \tau(s+1, r, \boldsymbol{t}, \overline{\boldsymbol{t}}), \\
\lambda^{-1} \tau & (s-1, r+1, \boldsymbol{t}, \overline{\boldsymbol{t}}+[\mu]) \tau\left(s+1, r+1, \boldsymbol{t}+\left[\lambda^{-1}\right], \overline{\boldsymbol{t}}\right) \\
& -\lambda^{-1} \tau\left(s, r+2, \boldsymbol{t}+\left[\lambda^{-1}\right], \overline{\boldsymbol{t}}+[\mu]\right) \tau(s, r, \boldsymbol{t}, \overline{\boldsymbol{t}}) \\
& =\mu^{-1} \tau\left(s, r+1, \boldsymbol{t}+\left[\lambda^{-1}\right], \overline{\boldsymbol{t}}\right) \tau(s, r+1, \boldsymbol{t}, \overline{\boldsymbol{t}}+[\mu]) \\
& -\mu^{-1} \tau\left(s, r+1, \boldsymbol{t}+\left[\lambda^{-1}\right], \overline{\boldsymbol{t}}+[\mu]\right) \tau(s, r+1, \boldsymbol{t}, \overline{\boldsymbol{t}}) .
\end{aligned}
$$

Actually, these calculations are meaningful even if the contour integrals are understood to be genuine algebraic operators that extract the coefficient of $z^{-1}$ from Laurent series. Thus we are led to the following conclusion:

Theorem 4. The bilinear equation (2.1) implies the Fay-like identities (4.1)-(4.6).

In the rest of this paper, (4.1)-(4.6) are referred to as "difference Fay identities". The structure of these Fay-like equations is similar to the difference Fay identities of the Toda hierarchy $[22,23,1]$, though the latter are three-term relations and given on a $1 D$ lattice.

\subsection{Relation to auxiliary linear problem}

We now show that the difference Fay identities are closely related to the auxiliary linear equations. To this end, let us rewrite the identities in the language of the wave functions as follows.

Theorem 5. The difference Fay identities (4.1)-(4.6) are equivalent to the system of the following four equations:

$$
\begin{gathered}
e^{-D(\lambda)} \Phi_{1}(s, r, \mu)+\lambda^{-1} \Phi_{1}(s+1, r, \mu)-\frac{e^{-D(\lambda)} \tau(s+1, r) / \tau(s+1, r)}{e^{-D(\lambda)} \tau(s, r) / \tau(s, r)} \Phi_{1}(s, r, \mu) \\
-\lambda^{-1} \frac{e^{-D(\lambda)} \tau(s+1, r) / \tau(s+1, r)}{e^{-D(\lambda)} \tau(s, r) / \tau(s+1, r+1)} e^{-D(\lambda)} \Phi_{2}(s+1, r, \mu)=0, \\
e^{D(\lambda)} \Phi_{2}(s, r, \mu)+\lambda^{-1} \Phi_{2}(s-1, r, \mu)-\frac{e^{D(\lambda)} \tau(s-1, r) / \tau(s-1, r)}{e^{D(\lambda)} \tau(s, r) / \tau(s, r)} \Phi_{2}(s, r, \mu) \\
-\lambda^{-1} \frac{e^{D(\lambda)} \tau(s-1, r) / \tau(s-1, r)}{e^{D(\lambda)} \tau(s, r) / \tau(s-1, r-1)} e^{D(\lambda)} \Phi_{1}(s-1, r, \mu)=0, \\
e^{-\bar{D}(\lambda)} \Phi_{1}(s, r, \mu)-\Phi_{1}(s, r, \mu)+\lambda \frac{e^{-\bar{D}(\lambda)} \tau(s+1, r) / \tau(s, r)}{e^{-\bar{D}(\lambda)} \tau(s, r) / \tau(s-1, r)} \Phi_{1}(s-1, r, \mu) \\
-\lambda \frac{e^{-\bar{D}(\lambda)} \tau(s+1, r) / \tau(s, r)}{e^{-\bar{D}(\lambda)} \tau(s, r) / \tau(s, r+1)} e^{-\bar{D}(\lambda)} \Phi_{2}(s+1, r, \mu)=0,
\end{gathered}
$$




$$
\begin{gathered}
e^{\bar{D}(\lambda)} \Phi_{2}(s, r, \mu)-\Phi_{2}(s, r, \mu)+\lambda \frac{e^{\bar{D}(\lambda)} \tau(s-1, r) / \tau(s, r)}{e^{\bar{D}(\lambda)} \tau(s, r) / \tau(s+1, r)} \Phi_{2}(s+1, r, \mu) \\
-\lambda \frac{e^{\bar{D}(\lambda)} \tau(s-1, r) / \tau(s, r)}{e^{\bar{D}(\lambda)} \tau(s, r) / \tau(s, r-1)} e^{\bar{D}(\lambda)} \Phi_{1}(s-1, r, \mu)=0
\end{gathered}
$$

for the four pairs $\Phi_{\alpha}=\Psi_{\alpha}, \Psi_{\alpha}^{*}, \bar{\Psi}_{\alpha}, \bar{\Psi}_{\alpha}^{*}(\alpha=1,2)$ of wave functions, where $D(z)$ and $\bar{D}(z)$ denote the differential operators

$$
D(z)=\sum_{n=1}^{\infty} \frac{z^{-n}}{n} \partial_{t_{n}}, \quad \bar{D}(z)=\sum_{n=1}^{\infty} \frac{z^{n}}{n} \partial_{\bar{t}_{n}} .
$$

Proof. One can derive the four difference Fay identities from (4.7)-(4.10) by straightforward calculations. Actually, this turns out to be largely redundant, namely, each difference Fay identity appears more than once while processing the twelve equations of (4.7)-(4.10). Nevertheless the calculations on the whole, are reversible, proving the converse simultaneously. Since the whole calculations are considerably lengthy, let us demonstrate it by deriving (4.1) and (4.2) from (4.7) and (4.8) for $\Phi_{\alpha}=\Psi_{\alpha}(\alpha=1,2)$; the other cases are fully parallel. Recall that $\Psi_{\alpha}$ 's can be expressed as

$$
\begin{aligned}
& \Psi_{1}(s, r, z)=\frac{\tau\left(s, r, \boldsymbol{t}-\left[z^{-1}\right], \overline{\boldsymbol{t}}\right)}{\tau(s, r, \boldsymbol{t}, \overline{\boldsymbol{t}})} z^{s+r} e^{\xi(\boldsymbol{t}, z)} \\
& \Psi_{2}(s, r, z)=\frac{\tau\left(s-1, r-1, \boldsymbol{t}-\left[z^{-1}\right], \overline{\boldsymbol{t}}\right)}{\tau(s, r, \boldsymbol{t}, \overline{\boldsymbol{t}})} z^{s+r-2} e^{\xi(\boldsymbol{t}, z)} .
\end{aligned}
$$

(4.7) thereby reads

$$
\begin{gathered}
e^{-D(\lambda)} \Psi_{1}(s, r, \mu)+\lambda^{-1} \Psi_{1}(s+1, r, \mu)-\frac{e^{-D(\lambda)} \tau(s+1, r) / \tau(s+1, r)}{e^{-D(\lambda)} \tau(s, r) / \tau(s, r)} \Psi_{1}(s, r, \mu) \\
-\lambda^{-1} \frac{e^{-D(\lambda)} \tau(s+1, r) / \tau(s+1, r)}{e^{-D(\lambda)} \tau(s, r) / \tau(s+1, r+1)} e^{-D(\lambda)} \Psi_{2}(s+1, r, \mu)=0 .
\end{gathered}
$$

Noting the identity

$$
e^{-D(\lambda)} e^{\xi(\boldsymbol{t}, \mu)}=(1-\mu / \lambda) e^{\xi(\boldsymbol{t}, \mu)},
$$

we thus obtain the equation

$$
\begin{aligned}
(1- & \left.\frac{\mu}{\lambda}\right) \frac{\tau\left(s, r, \boldsymbol{t}-\left[\lambda^{-1}\right]-\left[\mu^{-1}\right], \overline{\boldsymbol{t}}\right)}{\tau\left(s, r, \boldsymbol{t}-\left[\lambda^{-1}\right], \overline{\boldsymbol{t}}\right)}+\frac{\mu}{\lambda} \frac{\tau\left(s+1, r, \boldsymbol{t}-\left[\mu^{-1}\right], \overline{\boldsymbol{t}}\right)}{\tau(s+1, r, \boldsymbol{t}, \overline{\boldsymbol{t}})} \\
& -\frac{\tau\left(s+1, r, \boldsymbol{t}-\left[\lambda^{-1}\right], \overline{\boldsymbol{t}}\right) \tau\left(s, r, \boldsymbol{t}-\left[\mu^{-1}\right], \overline{\boldsymbol{t}}\right)}{\tau(s+1, r, \boldsymbol{t}, \overline{\boldsymbol{t}}) \tau\left(s, r, \boldsymbol{t}-\left[\lambda^{-1}\right], \overline{\boldsymbol{t}}\right)} \\
& -\frac{1}{\lambda \mu}\left(1-\frac{\mu}{\lambda}\right) \frac{\tau(s+1, r+1, \boldsymbol{t}, \overline{\boldsymbol{t}}) \tau\left(s, r-1, \boldsymbol{t}-\left[\lambda^{-1}\right]-\left[\mu^{-1}\right], \overline{\boldsymbol{t}}\right)}{\tau(s+1, r, \boldsymbol{t}, \overline{\boldsymbol{t}}) \tau\left(s, r, \boldsymbol{t}-\left[\mu^{-1}\right], \overline{\boldsymbol{t}}\right)}=0
\end{aligned}
$$

for the tau function. Rewriting this equation as

$$
\begin{aligned}
\tau\left(s, r, \boldsymbol{t}-\left[\lambda^{-1}\right]-\left[\mu^{-1}\right], \overline{\boldsymbol{t}}\right) \tau(s+1, r, \boldsymbol{t}, \overline{\boldsymbol{t}})+\frac{\mu}{\lambda-\mu} \tau\left(s+1, r, \boldsymbol{t}-\left[\mu^{-1}\right], \overline{\boldsymbol{t}}\right) \tau\left(s, r, \boldsymbol{t}-\left[\lambda^{-1}\right], \overline{\boldsymbol{t}}\right) \\
\quad-\frac{\lambda}{\lambda-\mu} \tau\left(s+1, r, \boldsymbol{t}-\left[\lambda^{-1}\right], \overline{\boldsymbol{t}}\right) \tau\left(s, r, \boldsymbol{t}-\left[\mu^{-1}\right], \overline{\boldsymbol{t}}\right) \\
\quad-\frac{1}{\lambda \mu} \tau(s+1, r+1, \boldsymbol{t}, \overline{\boldsymbol{t}}) \tau\left(s, r-1, \boldsymbol{t}-\left[\lambda^{-1}\right]-\left[\mu^{-1}\right], \overline{\boldsymbol{t}}\right)=0
\end{aligned}
$$


and shifting the variables as $\boldsymbol{t} \rightarrow \boldsymbol{t}+\left[\lambda^{-1}\right]+\left[\mu^{-1}\right]$ and $r \rightarrow r+1$, we arrive at (4.2). Let us now consider (4.8), namely,

$$
\begin{gathered}
e^{D(\lambda)} \Psi_{2}(s, r, \mu)+\lambda^{-1} \Psi_{2}(s-1, r, \mu)-\frac{e^{D(\lambda)} \tau(s-1, r) / \tau(s-1, r)}{e^{D(\lambda)} \tau(s, r) / \tau(s, r)} \Psi_{2}(s, r, \mu) \\
-\lambda^{-1} \frac{e^{D(\lambda)} \tau(s-1, r) / \tau(s-1, r)}{e^{D(\lambda)} \tau(s, r) / \tau(s-1, r-1)} e^{D(\lambda)} \Psi_{1}(s-1, r, \mu)=0 .
\end{gathered}
$$

This equation turns into the equation

$$
\begin{aligned}
\frac{\lambda}{\lambda-\mu} & \tau\left(s-1, r-1, \boldsymbol{t}+\left[\lambda^{-1}\right]-\left[\mu^{-1}\right], \overline{\boldsymbol{t}}\right) \tau(s-1, r, \boldsymbol{t}, \overline{\boldsymbol{t}}) \\
& +\frac{1}{\lambda \mu} \tau\left(s-2, r-1, \boldsymbol{t}-\left[\mu^{-1}\right], \overline{\boldsymbol{t}}\right) \tau\left(s, r, \boldsymbol{t}+\left[\lambda^{-1}\right], \overline{\boldsymbol{t}}\right) \\
& -\tau\left(s-1, r, \boldsymbol{t}+\left[\lambda^{-1}\right], \overline{\boldsymbol{t}}\right) \tau\left(s-1, r-1, \boldsymbol{t}-\left[\mu^{-1}\right], \overline{\boldsymbol{t}}\right) \\
& -\frac{\mu}{\lambda-\mu} \tau(s-1, r-1, \boldsymbol{t}, \overline{\boldsymbol{t}}) \tau\left(s-1, r, \boldsymbol{t}+\left[\lambda^{-1}\right]-\left[\mu^{-1}\right], \overline{\boldsymbol{t}}\right)=0
\end{aligned}
$$

for the tau function, and upon shifting the variables as $\boldsymbol{t} \rightarrow \boldsymbol{t}+\left[\mu^{-1}\right], s \rightarrow s+2$ and $r \rightarrow r+1$, reduces to (4.1). It will be obvious that these calculations are reversible.

Expanded in powers of $\lambda,(4.7)-(4.10)$ generate an infinite set of linear equations for the wave functions $\Phi_{\alpha}(s, r, \mu)$. As we show below, these linear equations are equivalent to the auxiliary linear equations (3.13) and (3.14). Thus (4.7)-(4.10) turn out to give a generating functional expression of these auxiliary linear equations.

Since the four equations (4.7)-(4.10) can be treated in the same manner, let us illustrate the calculations in the case of (4.7). Among the four terms in this equation, the first and fourth terms can be readily expanded by the identity

$$
e^{-D(\lambda)}=\sum_{n=0}^{\infty} h_{n}\left(-\tilde{\partial}_{t}\right) \lambda^{-n}, \quad \tilde{\partial}_{t}=\left(\partial_{t_{1}}, \frac{1}{2} \partial_{t_{2}}, \ldots, \frac{1}{n} \partial_{t_{n}}, \ldots\right) .
$$

As regards the coefficients of the second and third terms, we can rewrite them as

$$
\frac{e^{-D(\lambda)} \tau(s+1, r) / \tau(s+1, r)}{e^{-D(\lambda)} \tau(s, r) / \tau(s, r)}=1-\left(\log \frac{\tau(s+1, r)}{\tau(s, r)}\right)_{t_{1}} \lambda^{-1}+f_{2} \lambda^{-2}+f_{3} \lambda^{-3}+\cdots
$$

and

$$
\begin{aligned}
& \frac{e^{-D(\lambda)} \tau(s+1, r) / \tau(s+1, r)}{e^{-D(\lambda)} \tau(s, r) / \tau(s+1, r+1)} \\
& \quad=\frac{\tau(s+1, r+1)}{\tau(s, r)}\left(1-\left(\log \frac{\tau(s+1, r)}{\tau(s, r)}\right)_{t_{1}} \lambda^{-1}+f_{2} \lambda^{-2}+f_{3} \lambda^{-3}+\cdots\right),
\end{aligned}
$$

where $f_{n}$ 's denote the coefficients of the expansion

$$
\begin{aligned}
\exp \left(\left(e^{-D(\lambda)}-1\right) \log \frac{\tau(s+1, r)}{\tau(s, r)}\right) & =\exp \left(\sum_{n=1}^{\infty} \lambda^{-n} h_{n}\left(-\tilde{\partial}_{t}\right) \log \frac{\tau(s+1, r)}{\tau(s, r)}\right) \\
& =1+\sum_{n=1}^{\infty} f_{n} \lambda^{-n}
\end{aligned}
$$


Thus, expanding (4.7) in powers of $\lambda$, we obtain the equation

$$
\begin{gathered}
-\partial_{t_{1}} \Phi_{1}(s, r, \mu)+\left(e^{\partial_{s}}+\left(\log \frac{\tau(s+1, r)}{\tau(s, r)}\right)_{t_{1}}\right) \Phi_{1}(s, r, \mu) \\
-\frac{\tau(s+1, r+1)}{\tau(s, r)} e^{\partial_{s}} \Phi_{2}(s, r, \mu)=0
\end{gathered}
$$

from the $\lambda^{-1}$ terms and the equations

$$
\begin{aligned}
& h_{n+1}\left(-\tilde{\partial}_{t}\right) \Phi_{1}(s, r, \mu)-f_{n+1} \Phi_{1}(s, r, \mu) \\
& -\frac{\tau(s+1, r+1)}{\tau(s, r)} \sum_{m=0}^{n} f_{m} h_{n-m}\left(-\tilde{\partial}_{t}\right) e^{\partial_{s}} \Phi_{2}(s, r, \mu)=0
\end{aligned}
$$

for $n=1,2, \ldots$ from the $\lambda^{-n-1}$ terms.

In the same way, we can decompose (4.8), (4.9) and (4.10) into and the equations

$$
\begin{gathered}
\partial_{t_{1}} \Phi_{2}(s, r, \mu)+\left(e^{-\partial_{s}}-\left(\log \frac{\tau(s-1, r)}{\tau(s, r)}\right)_{t_{1}}\right) \Phi_{2}(s, r, \mu) \\
-\frac{\tau(s-1, r-1)}{\tau(s, r)} e^{-\partial_{s}} \Phi_{1}(s, r, \mu)=0 \\
-\partial_{\bar{t}_{1}} \Phi_{1}(s, r, \mu)+\frac{\tau(s+1, r) \tau(s-1, r)}{\tau(s, r)^{2}} e^{-\partial_{s}} \Phi_{1}(s, r, \mu) \\
-\frac{\tau(s+1, r) \tau(s, r+1)}{\tau(s, r)^{2}} e^{\partial_{s}} \Phi_{2}(s, r, \mu)=0, \\
\partial_{\bar{t}_{1}} \Phi_{2}(s, r, \mu)+\frac{\tau(s-1, r) \tau(s+1, r)}{\tau(s, r)^{2}} e^{\partial_{s}} \Phi_{2}(s, r, \mu) \\
-\frac{\tau(s-1, r) \tau(s, r-1)}{\tau(s, r)^{2}} e^{-\partial_{s}} \Phi_{1}(s, r, \mu)=0
\end{gathered}
$$

and the equations

$$
\begin{gathered}
h_{n+1}\left(\tilde{\partial}_{t}\right) \Phi_{2}(s, r, \mu)-g_{n+1} \Phi_{2}(r, s, \mu) \\
-\frac{\tau(s-1, r-1)}{\tau(s, r)} \sum_{m=0}^{n} g_{m} h_{n-m}\left(\tilde{\partial}_{t}\right) e^{-\partial_{s}} \Phi_{1}(s, r, \mu)=0, \\
h_{n+1}\left(-\tilde{\partial}_{\bar{t}}\right) \Phi_{1}(s, r, \mu)+\frac{\tau(s+1, r) \tau(s-1, r)}{\tau(s, r)^{2}} \bar{f}_{n} e^{-\partial_{s}} \Phi_{1}(s, r, \mu) \\
-\frac{\tau(s+1, r) \tau(s, r+1)}{\tau(s, r)^{2}} \sum_{m=0}^{n} \bar{f}_{m} h_{n-m}\left(-\tilde{\partial}_{\bar{t}}\right) e^{\partial_{s}} \Phi_{2}(s, r, \mu)=0, \\
h_{n+1}\left(\tilde{\partial}_{\bar{t}}\right) \Phi_{2}(s, r, \mu)+\frac{\tau(s+1, r) \tau(s-1, r)}{\tau(s, r)^{2}} \bar{g}_{n} e^{\partial_{s}} \Phi_{2}(s, r, \mu) \\
-\frac{\tau(s-1, r) \tau(s, r-1)}{\tau(s, r)^{2}} \sum_{m=0}^{n} \bar{g}_{m} h_{n-m}\left(\tilde{\partial}_{\bar{t}}\right) e^{-\partial_{s}} \Phi_{1}(s, r, \mu)=0
\end{gathered}
$$

for $n=1,2, \ldots$, where $g_{n}, \bar{f}_{n}$ and $\bar{g}_{n}$ are the coefficients of the expansion

$$
\exp \left(\left(e^{D(\lambda)}-1\right) \log \frac{\tau(s-1, r)}{\tau(s, r)}\right)=1+\sum_{n=1}^{\infty} g_{n} \lambda^{-n}
$$




$$
\begin{aligned}
& \exp \left(\left(e^{-\bar{D}(\lambda)}-1\right) \log \frac{\tau(s+1, r)}{\tau(s, r)}\right)=1+\sum_{n=1}^{\infty} \bar{f}_{n} \lambda^{n}, \\
& \exp \left(\left(e^{\bar{D}(\lambda)}-1\right) \log \frac{\tau(s-1, r)}{\tau(s, r)}\right)=1+\sum_{n=1}^{\infty} \bar{g}_{n} \lambda^{n} .
\end{aligned}
$$

Among these equations, the lowest ones (4.11), (4.13), (4.14) and (4.15) can be cast into a matrix form, which agrees with the lowest members (3.15) and (3.16) of (3.13) and (3.14). Though the other equations (4.12), (4.16), (4.17) and (4.18) do not take such an evolutionary form, they can be recursively converted to the form of (3.13) just as in the case of the DKP hierarchy [1]. Thus the auxiliary linear equations can be recovered from the difference Fay identities.

\section{Dispersionless limit}

\subsection{Dispersionless Hirota equations}

As in the case of the KP and Toda hierarchies [33], dispersionless limit is achieved by allowing the tau function to depend on a small parameter (Planck constant) $\hbar$ and assuming the "quasiclassical" behavior

$$
\tau_{\hbar}(s, r, \boldsymbol{t}, \overline{\boldsymbol{t}})=e^{\hbar^{-2} F(s, r, \boldsymbol{t}, \overline{\boldsymbol{t}})+O\left(\hbar^{-1}\right)} \quad(\hbar \rightarrow 0)
$$

of the rescaled tau function

$$
\tau_{\hbar}(s, r, \boldsymbol{t}, \overline{\boldsymbol{t}})=\tau\left(\hbar^{-1} s, \hbar^{-1} r, \hbar^{-1} \boldsymbol{t}, \hbar^{-1} \overline{\boldsymbol{t}}\right) .
$$

As we show below, the difference Fay identities for the rescaled tau function $\tau_{\hbar}(s, r, \boldsymbol{t}, \overline{\boldsymbol{t}})$ turn into differential equations for the $F$-function $F(s, r, \boldsymbol{t}, \overline{\boldsymbol{t}})$. Following the terminology commonly used in the literature, let us call those equations "dispersioness Hirota equations".

Let us first consider (4.1). Upon multiplying both hand sides by

$$
\frac{\tau(s+1, r+1, \boldsymbol{t}, \overline{\boldsymbol{t}})}{\tau\left(s+1, r+1, \boldsymbol{t}+\left[\lambda^{-1}\right], \overline{\boldsymbol{t}}\right) \tau\left(s+1, r+1, \boldsymbol{t}+\left[\mu^{-1}\right], \overline{\boldsymbol{t}}\right) \tau(s+1, r, \boldsymbol{t}, \overline{\boldsymbol{t}})},
$$

this equation turns into such a form as

$$
\begin{aligned}
& \frac{\tau\left(s+1, r+1, \boldsymbol{t}+\left[\lambda^{-1}\right]+\left[\mu^{-1}\right], \overline{\boldsymbol{t}}\right) \tau(s+1, r+1, \boldsymbol{t}, \overline{\boldsymbol{t}})}{\tau\left(s+1, r+1, \boldsymbol{t}+\left[\lambda^{-1}\right], \overline{\boldsymbol{t}}\right) \tau\left(s+1, r+1, \boldsymbol{t}+\left[\mu^{-1}\right], \overline{\boldsymbol{t}}\right)} \\
& =\frac{\lambda}{\lambda-\mu} \frac{\tau\left(s+1, r, \boldsymbol{t}+\left[\lambda^{-1}\right], \overline{\boldsymbol{t}}\right) \tau(s+1, r+1, \boldsymbol{t}, \overline{\boldsymbol{t}})}{\tau\left(s+1, r+1, \boldsymbol{t}+\left[\lambda^{-1}\right], \overline{\boldsymbol{t}}\right) \tau(s+1, r, \boldsymbol{t}, \overline{\boldsymbol{t}})} \\
& -\frac{\mu}{\lambda-\mu} \frac{\tau\left(s+1, r, \boldsymbol{t}+\left[\mu^{-1}\right], \overline{\boldsymbol{t}}\right) \tau(s+1, r+1, \boldsymbol{t}, \overline{\boldsymbol{t}})}{\tau\left(s+1, r+1, \boldsymbol{t}+\left[\mu^{-1}\right], \overline{\boldsymbol{t}}\right) \tau(s+1, r, \boldsymbol{t}, \overline{\boldsymbol{t}})} \\
& +\frac{1}{\lambda \mu} \frac{\tau\left(s+2, r+1, \boldsymbol{t}+\left[\lambda^{-1}\right]+\left[\mu^{-1}\right], \overline{\boldsymbol{t}}\right) \tau(s, r+1, \boldsymbol{t}, \overline{\boldsymbol{t}})}{\tau\left(s+1, r+1, \boldsymbol{t}+\left[\lambda^{-1}\right], \overline{\boldsymbol{t}}\right) \tau\left(s+1, r+1, \boldsymbol{t}+\left[\mu^{-1}\right], \overline{\boldsymbol{t}}\right)} \frac{\tau(s, r, \boldsymbol{t}, \overline{\boldsymbol{t}}) \tau(s+1, r+1, \boldsymbol{t}, \overline{\boldsymbol{t}})}{\tau(s, r+\boldsymbol{t}, \overline{\boldsymbol{t}}) \tau(s+1, r, \boldsymbol{t}, \overline{\boldsymbol{t}})} .
\end{aligned}
$$

We can rewrite both hand sides as

$$
\text { LHS }=\exp \left(\left(e^{D(\lambda)}-1\right)\left(e^{D(\mu)}-1\right) \log \tau(s+1, r+1)\right)
$$

and

$$
\mathrm{RHS}=\frac{\lambda}{\lambda-\mu} \exp \left(\left(e^{-\partial_{r}}-1\right)\left(e^{D(\lambda)}-1\right) \log \tau(s+1, r+1)\right)
$$




$$
\begin{aligned}
& -\frac{\mu}{\lambda-\mu} \exp \left(\left(e^{-\partial_{r}}-1\right)\left(e^{D(\mu)}-1\right) \log \tau(s+1, r+1)\right) \\
& +\frac{1}{\lambda \mu} \exp \left(\left(e^{\partial_{s}+D(\lambda)}-1\right)\left(e^{\partial_{s}+D(\mu)}-1\right) e^{-\partial_{s}} \log \tau(s+1, r+1)\right) \\
& \times \exp \left(\left(e^{-\partial_{r}}-1\right)\left(e^{-\partial_{s}}-1\right) \log \tau(s+1, r+1)\right) .
\end{aligned}
$$

Now rescale the variables $s, r, \boldsymbol{t}, \overline{\boldsymbol{t}}$ as

$$
s \rightarrow \hbar^{-1} s, \quad r \rightarrow \hbar^{-1} r, \quad \boldsymbol{t} \rightarrow \hbar^{-1} \boldsymbol{t}, \quad \overline{\boldsymbol{t}} \rightarrow \hbar^{-1} \overline{\boldsymbol{t}}
$$

The last equation thereby becomes an equation for the rescaled tau function $\tau_{\hbar}$, in which the derivatives are rescaled as

$$
\partial_{s} \rightarrow \hbar \partial_{s}, \quad \partial_{r} \rightarrow \hbar \partial_{r}, \quad D(z) \rightarrow \hbar D(z), \quad \bar{D}(z) \rightarrow \hbar \bar{D}(z)
$$

Under the quasi-classical ansatz (5.1), we can take the limit of this equation as $\hbar \rightarrow 0$. The outcome is the equation

$$
e^{D(\lambda) D(\mu) F}=\frac{\lambda e^{-\partial_{r} D(\lambda) F}-\mu e^{-\partial_{r} D(\mu) F}}{\lambda-\mu}+\frac{1}{\lambda \mu} e^{\left(\partial_{s}+D(\lambda)\left(\partial_{s}+D(\mu)\right) F+\partial_{r} \partial_{s} F\right.} .
$$

In much the same way, we can derive the following equations from the other differential Fay identities (4.2)-(4.6):

$$
\begin{aligned}
e^{D(\lambda) D(\mu) F}= & \frac{\lambda e^{-\partial_{s} D(\lambda) F}-\mu e^{-\partial_{s} D(\mu) F}}{\lambda-\mu}+\frac{1}{\lambda \mu} e^{\left(\partial_{r}+D(\lambda)\right)\left(\partial_{r}+D(\mu)\right) F+\partial_{r} \partial_{s} F}, \\
e^{\bar{D}(\lambda) \bar{D}(\mu) F}= & \frac{\lambda^{-1} e^{-\partial_{r} \bar{D}(\lambda) F}-\mu^{-1} e^{-\partial_{r} \bar{D}(\mu) F}}{\lambda^{-1}-\mu^{-1}}+\lambda \mu e^{\left(-\partial_{s}+\bar{D}(\lambda)\right)\left(-\partial_{s}+\bar{D}(\mu)\right) F-\partial_{r} \partial_{s} F}, \\
e^{\bar{D}(\lambda) \bar{D}(\mu) F}= & \frac{\lambda^{-1} e^{\partial_{s} \bar{D}(\lambda) F}-\mu^{-1} e^{\partial_{s} \bar{D}(\mu) F}}{\lambda^{-1}-\mu^{-1}}+\lambda \mu e^{\left(\partial_{r}+\bar{D}(\lambda)\right)\left(\partial_{r}+\bar{D}(\mu)\right) F-\partial_{r} \partial_{s} F} \\
e^{D(\lambda) \bar{D}(\mu) F}= & e^{-\left(\partial_{r}+\partial_{s}\right) \bar{D}(\mu) F}-\lambda \mu e^{-\left(\partial_{r} D(\lambda)+\partial_{s} \bar{D}(\mu)+\partial_{r} \partial_{s}\right) F} \\
& +\lambda \mu e^{\left(-\partial_{s}+D(\lambda)\right)\left(-\partial_{s}+\bar{D}(\mu)\right) F-\left(\partial_{r}+\partial_{s}\right) \partial_{s} F} \\
e^{D(\lambda) \bar{D}(\mu) F}= & 1-\frac{\mu}{\lambda} e^{\partial_{s}\left(\partial_{s}+D(\lambda)-\bar{D}(\mu)\right) F}+\frac{\mu}{\lambda} e^{\left(\partial_{r}+D(\lambda)\right)\left(\partial_{r}+\bar{D}(\mu)\right) F} .
\end{aligned}
$$

The six dispersionless Hirota equations can be divided to two distinct sets $\{(5.5),(5.7),(5.9)\}$ and $\{(5.4),(5.6),(5.8)\}$. The first set of equations may be thought of as analogues of the dispersionless Hirota equations of the Toda hierarchy $[22,24,25,26]$. The only difference is the presence of the last term on the right hand side of each equation. The second set of equations have no counterpart in the Toda hierarchy.

We can use (5.4), (5.6) and (5.8) to eliminate $e^{D(\lambda) D(\mu) F}, e^{\bar{D}(\lambda) \bar{D}(\mu) F}$ and $e^{D(\lambda) \bar{D}(\mu) F}$ from (5.5), (5.7) and (5.9). This leads to the following remarkable observation.

Theorem 6. The dispersionless Hirota equations (5.4)-(5.9) imply the following equations:

$$
\begin{aligned}
& \lambda\left(e^{-\partial_{s} D(\lambda) F}-e^{-\partial_{r} D(\lambda) F}\right)+\lambda^{-1}\left(e^{\partial_{s}\left(\partial_{r}+\partial_{s}+D(\lambda)\right) F}-e^{\partial_{r}\left(\partial_{r}+\partial_{s}+D(\lambda)\right) F}\right) \\
& \quad=\left(\partial_{r}-\partial_{s}\right) \partial_{t_{1}} F \\
& \quad \lambda^{-1}\left(e^{\partial_{s} \bar{D}(\lambda) F}-e^{-\partial_{r} \bar{D}(\lambda) F}\right)+\lambda\left(e^{-\partial_{s}\left(\partial_{r}-\partial_{s}+\bar{D}(\lambda)\right) F}-e^{\partial_{r}\left(\partial_{r}-\partial_{s}+\bar{D}(\lambda)\right) F}\right) \\
& \quad=\left(\partial_{r}+\partial_{s}\right) \partial_{\bar{t}_{1}} F \\
& \left(\partial_{r}+\partial_{s}\right) \partial_{\bar{t}_{1}} F=e^{-\partial_{r} \partial_{s} F}\left(\partial_{r}-\partial_{s}\right) \partial_{t_{1}} F
\end{aligned}
$$


Proof. We can rewrite (5.4) and (5.5) as

$$
\begin{aligned}
& e^{D(\lambda) D(\mu) F}\left(1-\frac{1}{\lambda \mu} e^{\partial_{s}\left(\partial_{r}+\partial_{s}+D(\lambda)+D(\mu)\right) F}\right)=\frac{\lambda e^{-\partial_{r} D(\lambda) F}-\mu e^{-\partial_{r} D(\lambda) F}}{\lambda-\mu}, \\
& e^{D(\lambda) D(\mu) F}\left(1-\frac{1}{\lambda \mu} e^{\partial_{r}\left(\partial_{r}+\partial_{s}+D(\lambda)+D(\mu)\right) F}\right)=\frac{\lambda e^{-\partial_{s} D(\lambda) F}-\mu e^{-\partial_{s} D(\mu) F}}{\lambda-\mu} .
\end{aligned}
$$

Eliminating $e^{D(\lambda) D(\mu) F}$ from these equations yields the equation

$$
\begin{aligned}
(1- & \left.\frac{1}{\lambda \mu} e^{\partial_{s}\left(\partial_{r}+\partial_{s}+D(\lambda)+D(\mu)\right) F}\right)\left(\lambda e^{-\partial_{s} D(\lambda) F}-\mu e^{-\partial_{s} D(\mu) F}\right) \\
& =\left(1-\frac{1}{\lambda \mu} e^{\partial_{r}\left(\partial_{r}+\partial_{s}+D(\lambda)+D(\mu)\right) F}\right)\left(\lambda e^{-\partial_{r} D(\lambda) F}-\mu e^{-\partial_{r} D(\mu) F}\right),
\end{aligned}
$$

which can be expanded as

$$
\begin{aligned}
& \lambda e^{-\partial_{s} D(\lambda) F}-\mu e^{-\partial_{s} D(\mu) F}+\lambda^{-1} e^{\partial_{s}\left(\partial_{r}+\partial_{s}+D(\lambda)\right) F}-\mu^{-1} e^{\partial_{s}\left(\partial_{r}+\partial_{s}+D(\mu)\right) F} \\
& \quad=\lambda e^{-\partial_{r} D(\lambda) F}-\mu e^{-\partial_{r} D(\mu) F}+\lambda^{-1} e^{\partial_{r}\left(\partial_{r}+\partial_{s}+D(\lambda)\right) F}-\mu^{-1} e^{\partial_{r}\left(\partial_{r}+\partial_{s}+D(\mu)\right) F} .
\end{aligned}
$$

We can separate $\lambda$-dependent and $\mu$-dependent terms to each side of the equation as

$$
\begin{aligned}
& \lambda\left(e^{-\partial_{s} D(\lambda) F}-e^{-\partial_{r} D(\lambda) F}\right)+\lambda^{-1}\left(e^{\partial_{s}\left(\partial_{r}+\partial_{s}+D(\lambda)\right) F}-e^{\partial_{r}\left(\partial_{r}+\partial_{s}+D(\lambda)\right) F}\right) \\
& \quad=\mu\left(e^{-\partial_{s} D(\mu) F}-e^{-\partial_{r} D(\mu) F}\right)+\mu^{-1}\left(e^{\partial_{s}\left(\partial_{r}+\partial_{s}+D(\mu)\right) F}-e^{\partial_{r}\left(\partial_{r}+\partial_{s}+D(\mu)\right) F}\right) .
\end{aligned}
$$

Therefore both hand sides of the last equation are independent of $\lambda$ and $\mu$. Letting $\lambda, \mu \rightarrow \infty$, we can readily see that this quantity is equal to $\left(\partial_{r}-\partial_{s}\right) \partial_{t_{1}} F$. Thus we obtain (5.10). In much the same way, we can derive (5.11) from (5.6) and (5.7). If we start from (5.8) and (5.9), we end up with the equation

$$
\begin{aligned}
& \mu^{-1}\left(e^{\partial_{s} \bar{D}(\mu) F}-e^{-\partial_{r} \bar{D}(\mu) F}\right)+\mu\left(e^{-\partial_{s}\left(\partial_{r}-\partial_{s}+\bar{D}(\mu)\right) F}-e^{\partial_{r}\left(\partial_{r}-\partial_{s}+\bar{D}(\mu)\right) F}\right) \\
& \quad=e^{-\partial_{r} \partial_{s} F}\left(\lambda\left(e^{-\partial_{s} D(\lambda) F}-e^{-\partial_{r} D(\lambda) F}\right)+\lambda^{-1}\left(e^{\partial_{s}\left(\partial_{r}+\partial_{s}+D(\lambda)\right) F}-e^{\partial_{r}\left(\partial_{r}+\partial_{s}+D(\lambda)\right) F}\right)\right) .
\end{aligned}
$$

By (5.10) and (5.11), this equation reduces to (5.12).

It is easy to see from this proof that the converse is also true. Namely, if (5.5), (5.7) and (5.9) holds, the other three dispersionless Hirota equations (5.4), (5.6) and (5.8) can be recovered from (5.10), (5.11) and (5.12). Thus we are led to the following conclusion.

Corollary 4. The dispersionless Hirota equations (5.4)-(5.9), can be reduced to the coupled system of the two sets of equations $\{(5.5),(5.7),(5.9)\}$ and $\{(5.10),(5.11),(5.12)\}$.

Thus the dispersionless Hirota equations of the Pfaff-Toda hierarchy can be reduced to two sets of equations that have quite different appearance and nature. Among the second set of equations, the last equation (5.12) is nothing but the dispersionless limit of (2.5). The other two equations (5.10) and (5.11) appear to be more mysterious. As it turns out below, they are related to special auxiliary linear equations for the wave functions.

\section{$5.2 \quad$ Elliptic spectral curve}

Let us examine (5.10), (5.11) and (5.12) in more detail. Substituting $\lambda \rightarrow z$, and applying (5.12) to the term on the right hand side of (5.11), we can rewrite (5.10) and (5.11) as

$$
z\left(e^{-\partial_{s} D(z) F}-e^{-\partial_{r} D(z) F}\right)+z^{-1}\left(e^{\partial_{s}\left(\partial_{r}+\partial_{s}+D(z)\right) F}-e^{\partial_{r}\left(\partial_{r}+\partial_{s}+D(z)\right) F}\right)=\left(\partial_{r}-\partial_{s}\right) \partial_{t_{1}} F,
$$


$z^{-1}\left(e^{\partial_{s} \bar{D}(z) F}-e^{-\partial_{r} \bar{D}(z) F}\right)+z\left(e^{-\partial_{s}\left(\partial_{r}-\partial_{s}+\bar{D}(z)\right) F}-e^{\partial_{r}\left(\partial_{r}-\partial_{s}+\bar{D}(z)\right) F}\right)=e^{-\partial_{r} \partial_{s} F}\left(\partial_{r}-\partial_{s}\right) \partial_{t_{1}} F$.

Actually, these two equations are equivalent to the set of three equations (5.10), (5.11) and (5.12), because letting $z \rightarrow 0$ in the second equation yields (5.12), and this enables one to recover (5.11) as well.

A clue of the subsequent consideration is to introduce the auxiliary functions

$$
\begin{aligned}
& S(z)=\xi(\boldsymbol{t}, z)+(s+r) \log z-D(z) F, \\
& \bar{S}(z)=\xi\left(\overline{\boldsymbol{t}}, z^{-1}\right)+(s-r) \log z+\partial_{s} F-\bar{D}(z) F .
\end{aligned}
$$

We can thereby rewrite these equations into the "Hamilton-Jacobi" form

$$
\begin{aligned}
& e^{\partial_{r} S(z)}=e^{\partial_{s} S(z)}+\left(\partial_{s}-\partial_{r}\right) \partial_{t_{1}} F+e^{\partial_{s}\left(\partial_{r}+\partial_{s}\right) F} e^{-\partial_{s} S(z)}-e^{\partial_{r}\left(\partial_{r}+\partial_{s}\right) F} e^{-\partial_{r} S(z)}, \\
& e^{\partial_{r} \bar{S}(z)}=e^{\partial_{s} \bar{S}(z)}+\left(\partial_{s}-\partial_{r}\right) \partial_{t_{1}} F+e^{\partial_{s}\left(\partial_{r}+\partial_{s}\right) F} e^{-\partial_{s} \bar{S}(z)}-e^{\partial_{r}\left(\partial_{r}+\partial_{s}\right) F} e^{-\partial_{r} \bar{S}(z)}
\end{aligned}
$$

These equations imply that the exponentiated gradient vectors $\left(e^{\partial_{s} S(z)}, e^{\partial_{r} S(z)}\right)$ and $\left(e^{\partial_{s} \bar{S}(z)}\right.$, $\left.e^{\partial_{r} \bar{S}(z)}\right)$ of the two $S$-functions on the $(s, r)$ plane satisfy the same algebraic equation

$$
Q=P+\left(\partial_{s}-\partial_{r}\right) \partial_{t_{1}} F+e^{\partial_{s}\left(\partial_{r}+\partial_{s}\right) F} P^{-1}-e^{\partial_{r}\left(\partial_{r}+\partial_{s}\right) F} Q^{-1}
$$

This equation defines a curve of genus one on the $(P, Q)$ plane.

We now argue that this equation can be identified with the characteristic equation of a set of auxiliary linear equations. In other words, this curve is indeed a "spectral curve".

The functions $S(z)$ and $\bar{S}(z)$ may be thought of as the phase functions of the quasi-classical (WKB) ansatz

$$
\Psi_{1}(z)=e^{\hbar^{-1} S(z)+O(1)}, \quad \bar{\Psi}_{1}(z)=e^{\hbar^{-1} \bar{S}(z)+O(1)} \quad(\hbar \rightarrow 0)
$$

of the wave functions $\Psi_{1}(z)$ and $\bar{\Psi}_{1}(z)$. Consequently, it will be natural to expect that (5.13) and (5.14) are Hamilton-Jacobi equations of linear equations of the form

$$
e^{\hbar \partial_{r}} \Phi_{1}(z)=\left(e^{\hbar \partial_{s}}+a+b e^{-\hbar \partial_{s}}+c e^{-\hbar \partial_{r}}\right) \Phi_{1}(z)
$$

for $\Phi_{1}(z)=\Psi_{1}(z), \bar{\Psi}_{1}(z)$, and that the coefficients have the quasi-classical limit

$$
\lim _{\hbar \rightarrow 0} a=\left(\partial_{s}-\partial_{r}\right) F, \quad \lim _{\hbar \rightarrow 0} b=e^{\partial_{s}\left(\partial_{r}+\partial_{s}\right) F}, \quad \lim _{\hbar \rightarrow 0} c=-e^{\partial_{r}\left(\partial_{r}+\partial_{s}\right) F}
$$

so that (5.15) can be interpreted as the characteristic equation of (5.16).

We can indeed derive such linear equations from (3.12). To simplify notations, let us consider the case where $\hbar=1$; one can readily transfer to the $\hbar$-dependent setup of the quasi-classical ansatz by rescaling the variables and derivative as (5.2) and (5.3). Written in terms of components, (3.12) consists of the linear equations

$$
e^{\partial_{r}} \Phi_{1}(z)=A \Phi_{1}(z)+B \Phi_{2}(z), \quad e^{\partial_{r}} \Phi_{2}(z)=C \Phi_{1}(z)
$$

for the four pairs $\Phi_{\alpha}(z)=\Psi_{\alpha}(z), \Psi_{\alpha}^{*}(z), \bar{\Psi}_{\alpha}(z), \bar{\Psi}_{\alpha}^{*}(z)(\alpha=1,2)$, where

$$
\begin{aligned}
& A=e^{\partial_{s}}+\left(\log \frac{\tau(s+1, r)}{\tau(s, r+1)}\right)_{t_{1}}+\frac{\tau(s+1, r+1) \tau(s-1, r)}{\tau(s, r+1) \tau(s, r)} e^{-\partial_{s}}, \\
& B=-\frac{\tau(s+1, r+1)}{\tau(s, r)} e^{\partial_{s}}, \quad C=\frac{\tau(s-1, r)}{\tau(s, r+1)} e^{-\partial_{s}} .
\end{aligned}
$$


The second equation implies that $\Phi_{1}(z)$ and $\Phi_{2}(z)$ are connected by the relation

$$
\Phi_{2}(z)=e^{-\partial_{r}} C \Phi_{1}(z)=\frac{\tau(s-1, r-1)}{\tau(s, r)} e^{-\partial_{s}-\partial_{r}} \Phi_{1}(z),
$$

which one can see from the definition (3.1) and (3.2) of the wave functions as well. Using this relation, we can eliminate $\Phi_{2}(z)$ from the first equation and obtain the equation

$$
e^{\partial_{r}} \Phi_{1}(z)=\left(A+B e^{-\partial_{r}} C\right) \Phi_{1}(z)
$$

After some algebra, this equation boils down to (5.16). Moreover, though we omit details, the coefficients turn out to take such a form as

$$
\begin{aligned}
& a=\left(\log \frac{\tau(s+1, r)}{\tau(s, r+1)}\right)_{t_{1}}, \quad b=\frac{\tau(s+1, r+1) \tau(s-1, r)}{\tau(s, r+1) \tau(s, r)}, \\
& c=-\frac{\tau(s+1, r+1) \tau(s, r-1)}{\tau(s+1, r) \tau(s, r)} .
\end{aligned}
$$

It is easy to see that these coefficients do have the anticipated quasi-classical limit.

The other auxiliary linear equations (3.13) and (3.14), too, can be converted to scalar linear equations of the form

$$
\partial_{t_{n}} \Phi_{1}(z)=\left(A_{n}+B_{n} e^{-\partial_{r}} C\right) \Phi_{1}(z), \quad \partial_{\bar{t}_{n}} \Phi_{1}(z)=\left(\bar{A}_{n}+\bar{B}_{n} e^{-\partial_{r}} C\right) \Phi_{1}(z) .
$$

Note that the $2 D$ difference operators

$$
K_{n}\left(e^{\partial_{s}}, e^{\partial_{r}}\right)=A_{n}+B_{n} e^{-\partial_{r}} C, \quad \bar{K}_{n}\left(e^{\partial_{s}}, e^{\partial_{r}}\right)=\bar{A}_{n}+\bar{B}_{n} e^{-\partial_{r}} C
$$

show up on the right hand sides of these equations. Consequently, the S-functions $S(z)$ and $\bar{S}(z)$ satisfy the Hamilton-Jacobi equations

$$
\begin{array}{ll}
\partial_{t_{n}} S(z)=K_{n}\left(e^{\partial_{s} S(z)}, e^{\partial_{r} S(z)}\right), & \partial_{\bar{t}_{n}} S(z)=\bar{K}_{n}\left(e^{\partial_{s} S(z)}, e^{\partial_{r} S(z)}\right), \\
\partial_{t_{n}} \bar{S}(z)=K_{n}\left(e^{\partial_{s} \bar{S}(z)}, e^{\partial_{r} \bar{S}(z)}\right), & \partial_{\bar{t}_{n}} \bar{S}(z)=\bar{K}_{n}\left(e^{\partial_{s} \bar{S}(z)}, e^{\partial_{r} \bar{S}(z)}\right) .
\end{array}
$$

Those Hamilton-Jacobi equations and the constraints (5.13) and (5.14) may be thought of as defining "quasi-classical deformations" [28, 29] of the spectral curve (5.15).

\subsection{Comparison with DKP hierarchy}

Let us compare the foregoing results with the case of the DKP hierarchy [1, 27].

The situation of the DKP hierarchy is similar to the KP hierarchy rather than the Toda hierarchy. The role of the difference Fay identities (4.1)-(4.6) are played by the differential Fay identities

$$
\begin{aligned}
& \frac{\tau\left(r, \boldsymbol{t}+\left[\lambda^{-1}\right]+\left[\mu^{-1}\right]\right) \tau(r, \boldsymbol{t})}{\tau\left(r, \boldsymbol{t}+\left[\lambda^{-1}\right]\right) \tau\left(r, \boldsymbol{t}+\left[\mu^{-1}\right]\right)}-\frac{1}{\lambda^{2} \mu^{2}} \frac{\tau\left(r+1, \boldsymbol{t}+\left[\lambda^{-1}\right]+\left[\mu^{-1}\right]\right) \tau(r-1, \boldsymbol{t})}{\tau\left(r, \boldsymbol{t}+\left[\lambda^{-1}\right]\right) \tau\left(r, \boldsymbol{t}+\left[\mu^{-1}\right]\right)} \\
& \quad=1-\frac{1}{\lambda-\mu} \partial_{t_{1}} \log \frac{\tau\left(r, \boldsymbol{t}+\left[\lambda^{-1}\right]\right)}{\tau\left(r, \boldsymbol{t}+\left[\mu^{-1}\right]\right)}, \\
& \frac{\lambda^{2}}{\lambda-\mu} \frac{\tau\left(r-1, \boldsymbol{t}+\left[\lambda^{-1}\right]\right) \tau\left(r, \boldsymbol{t}+\left[\mu^{-1}\right]\right)}{\tau\left(r, \boldsymbol{t}+\left[\lambda^{-1}\right]+\left[\mu^{-1}\right]\right) \tau(r-1, \boldsymbol{t})}-\frac{\mu^{2}}{\lambda-\mu} \frac{\tau\left(r, \boldsymbol{t}+\left[\lambda^{-1}\right]\right) \tau\left(r-1, \boldsymbol{t}+\left[\mu^{-1}\right]\right)}{\tau\left(r, \boldsymbol{t}+\left[\lambda^{-1}\right]+\left[\mu^{-1}\right]\right) \tau(r-1, \boldsymbol{t})} \\
& \quad=\lambda+\mu-\partial_{t_{1}} \log \frac{\tau\left(r, \boldsymbol{t}+\left[\lambda^{-1}\right]+\left[\mu^{-1}\right]\right)}{\tau(r-1, \boldsymbol{t})} .
\end{aligned}
$$


In the dispersionless limit, they turn into the dispersionless Hirota equations

$$
\begin{aligned}
& e^{D(\lambda) D(\mu) F}-\lambda^{-2} \mu^{-2} e^{\left(\partial_{r}+D(\lambda)\right)\left(\partial_{r}+D(\mu) F\right.}=1-\frac{\partial_{t_{1}}(D(\lambda)-D(\mu)) F}{\lambda-\mu} \\
& \frac{\lambda^{2}}{\lambda-\mu} e^{-D(\lambda)\left(\partial_{r}+D(\mu)\right) F}-\frac{\mu^{2}}{\lambda-\mu} e^{-\left(\partial_{r}+D(\lambda)\right) D(\mu) F}=\lambda+\mu-\partial_{t_{1}}\left(\partial_{r}+D(\lambda)+D(\mu)\right) F
\end{aligned}
$$

for the $F$-function $F(r, \boldsymbol{t})$. The first equation (5.21) resembles the dispersionless Hirota equation of the KP hierarchy.

It is convenient to introduce the $S$-function

$$
S(z)=\xi(\boldsymbol{t}, z)+2 r \log z-D(z) F
$$

at this state. We can thereby rewrite (5.21) and (5.22) as

$$
\begin{aligned}
& e^{D(\lambda) D(\mu) F}\left(1-e^{\partial_{r}^{2} F-\partial_{r} S(\lambda)-\partial_{r} S(\mu)}\right)=\frac{\partial_{1} S(\lambda)-\partial_{1} S(\mu)}{\lambda-\mu} \\
& \frac{e^{-D(\lambda) D(\mu) F}}{\lambda-\mu}\left(e^{\partial_{r} S(\lambda)}-e^{\partial_{r} S(\mu)}\right)=\partial_{t_{1}} S(\lambda)+\partial_{t_{1}} S(\mu)-\partial_{r} \partial_{t_{1}} F
\end{aligned}
$$

and eliminate $e^{D(\lambda) D(\mu) F}$ from these two equations. This leads to the equality

$$
\begin{aligned}
& \left(\partial_{t_{1}} S(\lambda)\right)^{2}-\left(\partial_{t_{1}} \partial_{r} F\right)\left(\partial_{t_{1}} S(\lambda)\right)-e^{\partial_{r} S(\lambda)}-e^{\partial_{r}^{2} F-\partial_{r} S(\lambda)} \\
& \quad=\left(\partial_{t_{1}} S(\mu)\right)^{2}-\left(\partial_{t_{1}} \partial_{r} F\right)\left(\partial_{t_{1}} S(\mu)\right)-e^{\partial_{r} S(\mu)}-e^{\partial_{r}^{2} F-\partial_{r} S(\mu)}
\end{aligned}
$$

hence both hand sides are independent of $\lambda$ and $\mu$. Letting $\lambda, \mu \rightarrow \infty$, we can determine this quantity explicitly. Thus we obtain the equation

$$
\begin{gathered}
\left(\partial_{t_{1}} S(z)\right)^{2}-\left(\partial_{t_{1}} \partial_{r} F\right)\left(\partial_{t_{1}} S(z)\right)-e^{\partial_{r} S(z)}-e^{\partial_{r}^{2} F-\partial_{r} S(z)} \\
=-2 \partial_{t_{1}}^{2} F+\frac{1}{2} \partial_{t_{2}} \partial_{r} F-\frac{1}{2}\left(\partial_{t_{1}} \partial_{r} F\right)^{2}
\end{gathered}
$$

In other words, the partially exponentiated gradient vector $\left(\partial_{t_{1}} S(z), e^{\partial_{r} S(z)}\right)$ of the $S$-function satisfies the algebraic equation

$$
p^{2}-\left(\partial_{t_{1}} \partial_{r} F\right) p-Q-e^{\partial_{r}^{2} F} Q^{-1}=-2 \partial_{t_{1}}^{2} F+\frac{1}{2} \partial_{t_{2}} \partial_{r} F-\frac{1}{2}\left(\partial_{t_{1}} \partial_{r} F\right)^{2}
$$

of an elliptic curve on the $(p, Q)$ plane. This curve was studied by Kodama and Pierce [27] as an analogue of the spectral curve of the $1 D$ Toda hierarchy.

(5.23) and (5.24) are the Hamilton-Jacobi and characteristic equations of a linear equation of the form

$$
e^{\hbar \partial_{r}} \Phi_{1}(z)=\left(\hbar^{2} \partial_{t_{1}}^{2}+\hbar a \partial_{t_{1}}+b+c e^{-\hbar \partial_{r}}\right) \Phi_{1}(z) .
$$

We can derive this equation from one of auxiliary linear equations as follows. Let us again consider the case where $\hbar=1$. As in the case of the Pfaff-Toda hierarchy, we can eliminate $\Psi_{2}(z)$ and $\Psi_{2}^{*}(z)$ from the auxiliary linear equation (A.7) by the relation

$$
\Phi_{2}(z)=\frac{\tau(r-1)}{\tau(r)} e^{-\partial_{r}} \Phi_{1}(z)
$$


that holds for $\Phi_{\alpha}(z)=\Psi_{\alpha}(z), \Psi_{\alpha}^{*}(z)(\alpha=1,2)$. The matrix equation (A.7) thereby reduces to a scalar equation of the form (5.25) for $\Phi_{1}(z)=\Psi_{1}(z), \Psi_{1}^{*}(z)$. The coefficients $a, b, c$ can be determined explicitly as

$$
\begin{aligned}
a= & -\left(\log \frac{\tau(r+1)}{\tau(r)}\right)_{t_{1}}, \quad c=-\frac{\tau(r+1) \tau(r-1)}{\tau(r)^{2}}, \\
b= & \frac{\tau(r+1)_{t_{1} t_{1}}-\tau(r+1)_{t_{2}}}{2 \tau(r+1)}-\frac{\tau(r)_{t_{1} t_{1}}-\tau(r)_{t_{2}}}{2 \tau(r)} \\
& -\left(\log \frac{\tau(r+1)}{\tau(r)}\right)_{t_{1}}(\log \tau(r))_{t_{1}}+2(\log \tau(r))_{t_{1} t_{1}} .
\end{aligned}
$$

Rescaling the variables as (5.2), one can correctly recover the coefficients of (5.23) and (5.24) in the quasi-classical limit:

$$
\lim _{\hbar \rightarrow 0} a=-\partial_{t_{1}} \partial_{r} F, \quad \lim _{\hbar \rightarrow 0} c=-e^{\partial_{r}^{2} F}, \quad \lim _{\hbar \rightarrow 0} b=-\frac{1}{2} \partial_{t_{2}} \partial_{r} F+\frac{1}{2}\left(\partial_{t_{1}} \partial_{r} F\right)^{2}+2 \partial_{t_{1}}^{2} F .
$$

\section{Conclusion}

We have thus obtained the following equations that characterize various aspects of the PfaffToda hierarchy:

- the algebraic constraints (3.6), (3.7) and the evolution equations (3.8), (3.10), (3.11) of the dressing operators,

- the auxiliary linear equations (3.12)-(3.14),

- the difference Fay identities (4.1)-(4.6),

- the generating functional expression (4.7)-(4.10) of the auxiliary linear equations (3.13) and (3.14),

- the dispersionless Hirota equations, (5.4)-(5.9),

- the defining equation (5.15) of the elliptic spectral curve,

- the Hamilton-Jacobi equations (5.13), (5.14), (5.17) and (5.18).

All these equations have counterparts in the DKP hierarchy. We have thus demonstrated that the Pfaff-Toda hierarchy is indeed a Toda version of the DKP hierarchy (or a Pfaffian version of the Toda hierarchy).

Actually, this is not the end of the story. Let us note a few open problems.

Firstly, although the other auxiliary linear equations (3.13) and (3.14) have been encoded to the difference Fay identities, the status of the remaining equation (3.12) is still obscure. Since its counterpart in the dispersionless limit are (5.13) and (5.14), and these equations are obtained from the dispersionless Hirota equations, it seems likely that (3.12), too, can be derived from the difference Fay identities. Unfortunately, we have been unable to find a direct proof. If this conjecture is true, it leads to an important conclusion that the difference Fay identities are, on the whole, equivalent to the Pfaff-Toda hierarchy itself, as it is indeed the case for the KP hierarchy $[1,33]$ and the Toda hierarchy [23].

Secondly, very little is known about special solutions of the Pfaff-Toda hierarchy. Of course one can freely generate solutions by the fermionic formula. Finding an interesting class of solutions is, however, a nontrivial problem. A possible strategy will be to seek, again, for analogy with the DKP hierarchy. 


\section{A Auxiliary linear problem of DKP hierarchy}

The DKP hierarchy has a discrete variable $r$ and a set of continuous variables $\boldsymbol{t}=\left(t_{1}, t_{2}, \ldots\right)$. The Tau function $\tau=\tau(r, \boldsymbol{t})$ satisfies the bilinear equation

$$
\begin{aligned}
& \oint \frac{d z}{2 \pi i} z^{2 r^{\prime}-2 r} e^{\xi\left(\boldsymbol{t}^{\prime}-\boldsymbol{t}, z\right)} \tau\left(r^{\prime}, \boldsymbol{t}^{\prime}-\left[z^{-1}\right]\right) \tau\left(r, \boldsymbol{t}+\left[z^{-1}\right]\right) \\
& \quad+\oint \frac{d z}{2 \pi i} z^{2 r-2 r^{\prime}-4} e^{\xi\left(\boldsymbol{t}-\boldsymbol{t}^{\prime}, z\right)} \tau\left(r^{\prime}+1, \boldsymbol{t}^{\prime}+\left[z^{-1}\right]\right) \tau\left(r-1, \boldsymbol{t}-\left[z^{-1}\right]\right)=0 .
\end{aligned}
$$

This bilinear equation can be converted to the Hirota form

$$
\begin{aligned}
\sum_{n=0}^{\infty} h_{n}(-2 \boldsymbol{a}) h_{n+2 r^{\prime}-2 r+1}\left(\tilde{D}_{\boldsymbol{t}}\right) e^{\left\langle\boldsymbol{a}, D_{\boldsymbol{t}}\right\rangle} \tau(r, \boldsymbol{t}) \cdot \tau\left(r^{\prime}, \boldsymbol{t}\right) \\
\quad+\sum_{n=0}^{\infty} h_{n}(2 \boldsymbol{a}) h_{n+2 r-2 r^{\prime}-3}\left(-\tilde{D}_{\boldsymbol{t}}\right) e^{\left\langle\boldsymbol{a}, D_{\boldsymbol{t}}\right\rangle} \tau(r-1, \boldsymbol{t}) \cdot \tau\left(r^{\prime}+1, \boldsymbol{t}\right)=0
\end{aligned}
$$

with an infinite set of arbitrary constants $\boldsymbol{a}=\left(a_{1}, a_{2}, \ldots\right)$. This is a generating functional expression of an infinite number of Hirota equations. The differential Fay identities (5.19) and (5.20) can be derived by differentiating the bilinear equation by $t_{1}^{\prime}$ and specializing the variables as follows:

1) $\boldsymbol{t}^{\prime}=\boldsymbol{t}+\left[\lambda^{-1}\right]+\left[\mu^{-1}\right], r^{\prime}=r$

2) $\boldsymbol{t}^{\prime}=\boldsymbol{t}+\left[\lambda^{-1}\right]-\left[\mu^{-1}\right], r^{\prime}=r-1$.

The bilinear equation (A.1) of the tau function leads to a set of bilinear equations for the wave functions

$$
\begin{aligned}
& \Psi_{1}(r, \boldsymbol{t}, z)=z^{2 r} e^{\xi(\boldsymbol{t}, z)} \frac{\tau\left(r, \boldsymbol{t}-\left[z^{-1}\right]\right)}{\tau(r, \boldsymbol{t})} \\
& \Psi_{2}(r, \boldsymbol{t}, z)=z^{2 r-2} e^{\xi(\boldsymbol{t}, z)} \frac{\tau\left(r-1, \boldsymbol{t}-\left[z^{-1}\right]\right)}{\tau(r, \boldsymbol{t})} \\
& \Psi_{1}^{*}(r, \boldsymbol{t}, z)=z^{-2 r-2} e^{-\xi(\boldsymbol{t}, z)} \frac{\tau\left(r+1, \boldsymbol{t}+\left[z^{-1}\right]\right)}{\tau(r, \boldsymbol{t})} \\
& \Psi_{2}^{*}(r, \boldsymbol{t}, z)=z^{-2 r} e^{-\xi(\boldsymbol{t}, z)} \frac{\tau\left(r, \boldsymbol{t}+\left[z^{-1}\right]\right)}{\tau(r, \boldsymbol{t})}
\end{aligned}
$$

These bilinear equations can be cast into the matrix form

$$
\oint \frac{d z}{2 \pi i} \Psi_{2 \times 2}\left(r^{\prime}, \boldsymbol{t}^{\prime}, z\right){ }^{\mathrm{t}} \Psi_{2 \times 2}^{*}(r, \boldsymbol{t}, z)=0,
$$

where

$$
\Psi_{2 \times 2}(r, \boldsymbol{t}, z)=\left(\begin{array}{ll}
\Psi_{1}(r, \boldsymbol{t}, z) & \Psi_{1}^{*}(r, \boldsymbol{t}, z) \\
\Psi_{2}(r, \boldsymbol{t}, z) & \Psi_{2}^{*}(r, \boldsymbol{t}, z)
\end{array}\right), \quad \Psi_{2 \times 2}^{*}(r, \boldsymbol{t}, z)=\left(\begin{array}{cc}
\Psi_{1}^{*}(r, \boldsymbol{t}, z) & \Psi_{1}(r, \boldsymbol{t}, z) \\
\Psi_{2}^{*}(r, \boldsymbol{t}, z) & \Psi_{2}(r, \boldsymbol{t}, z)
\end{array}\right) .
$$

The wave functions are associated with dressing operators of the form

$$
\begin{array}{ll}
W_{1}=1+\sum_{n=1}^{\infty} w_{1 n} \partial_{t_{1}}^{-n}, & V_{1}=\sum_{n=0}^{\infty} v_{1 n}\left(-\partial_{t_{1}}\right)^{-n-2}, \\
W_{2}=\sum_{n=0}^{\infty} w_{2 n} \partial_{t_{1}}^{-n-2}, & V_{2}=1+\sum_{n=1}^{\infty} v_{2 n}\left(-\partial_{t_{1}}\right)^{-n} .
\end{array}
$$


The coefficients are determined by Laurent expansion of the tau-quotient in the wave functions as

$$
\begin{array}{ll}
\frac{\tau\left(r, \boldsymbol{t}-\left[z^{-1}\right]\right)}{\tau(r, \boldsymbol{t})}=1+\sum_{n=1}^{\infty} w_{1 n} z^{-n}, & \frac{\tau\left(r+1, \boldsymbol{t}+\left[z^{-1}\right]\right)}{\tau(r, \boldsymbol{t})}=\sum_{n=0}^{\infty} v_{1 n} z^{-n}, \\
\frac{\tau\left(r-1, \boldsymbol{t}-\left[z^{-1}\right]\right)}{\tau(r, \boldsymbol{t})}=\sum_{n=0}^{\infty} w_{2 n} z^{-n}, & \frac{\tau\left(r, \boldsymbol{t}+\left[z^{-1}\right]\right)}{\tau(r, \boldsymbol{t})}=1+\sum_{n=1}^{\infty} v_{2 n} z^{-n} .
\end{array}
$$

The wave functions are thereby expressed as

$$
\Psi_{\alpha}(r, \boldsymbol{t}, z)=W_{\alpha} z^{2 r} e^{\xi(\boldsymbol{t}, z)}, \quad \Psi_{\alpha}^{*}(r, \boldsymbol{t}, z)=V_{\alpha} z^{-2 r} e^{-\xi(\boldsymbol{t}, z)}
$$

for $\alpha=1,2$.

Various equations for the dressing operators can be derived from this bilinear equation. A technical clue is an analogue of (3.4) for pseudo-differential operators [30, 31, 32]. For a pair of pseudo-differential operators of the form

$$
P=\sum_{n=-\infty}^{\infty} p_{n}(x) \partial_{x}^{n}, \quad Q=\sum_{n=-\infty}^{\infty} q_{n}(x) \partial_{x}^{n}
$$

let $\Psi(x, z)$ and $\Phi(x, z)$ denote the wave functions

$$
\Psi(x, z)=P e^{x z}=\sum_{n=-\infty}^{\infty} p_{n}(x) z^{n} e^{x z}, \quad \Phi(x, z)=Q e^{-x z}=\sum_{n=-\infty}^{\infty} q_{n}(x)(-z)^{n} e^{-x z} .
$$

Moreover, let $P^{*}$ denote the formal adjoint

$$
P^{*}=\sum_{n=-\infty}^{\infty}\left(-\partial_{x}\right)^{n} p_{n}(x)
$$

Then one has the identity

$$
\oint \frac{d z}{2 \pi i} \Psi\left(x^{\prime}, z\right) \Phi(x, z)=\sum_{k=0}^{\infty}\left(P Q^{*}\right)_{-k-1} \frac{\left(x^{\prime}-x\right)^{k}}{k !}=-\sum_{k=0}^{\infty}\left(Q P^{*}\right)_{-k-1} \frac{\left(x-x^{\prime}\right)^{k}}{k !}
$$

where ( $)_{-k-1}$ stands for the coefficient of $\partial_{x}^{-k-1}$ of a pseudo-differential operator.

With the aid of this formula (A.3), one can derive the algebraic constraint

$$
\left(\begin{array}{ll}
W_{1} & V_{1} \\
W_{2} & V_{2}
\end{array}\right)^{*}=\left(\begin{array}{cc}
0 & 1 \\
-1 & 0
\end{array}\right)\left(\begin{array}{ll}
W_{1} & V_{1} \\
W_{2} & V_{2}
\end{array}\right)^{-1}\left(\begin{array}{cc}
0 & -1 \\
1 & 0
\end{array}\right)
$$

the discrete evolution equation

$$
\left(\begin{array}{ll}
W_{1}(r+1) & V_{1}(r+1) \\
W_{2}(r+1) & V_{2}(r+1)
\end{array}\right)\left(\begin{array}{cc}
\partial_{t_{1}}^{2} & 0 \\
0 & \partial_{t_{1}}^{-2}
\end{array}\right)=\left(\begin{array}{cc}
A & B \\
C & 0
\end{array}\right)\left(\begin{array}{ll}
W_{1} & V_{1} \\
W_{2} & V_{2}
\end{array}\right)
$$

and the continuous evolution equations

$$
\left(\begin{array}{ll}
W_{1, t_{n}}+W_{1} \partial_{t_{1}} & V_{1, t_{n}}-V_{2}\left(-\partial_{t_{1}}\right)^{n} \\
W_{2, t_{n}}+W_{2} \partial_{t_{1}} & V_{2, t_{n}}-V_{2}\left(-\partial_{t_{1}}\right)^{n}
\end{array}\right)=\left(\begin{array}{cc}
A_{n} & B_{n} \\
C_{n} & D_{n}
\end{array}\right)\left(\begin{array}{cc}
W_{1} & V_{1} \\
W_{2} & V_{2}
\end{array}\right)
$$


from the bilinear equation (A.2) of the wave functions. Here $A, B, C$ are differential operators of the form

$$
A=\partial_{t_{1}}^{2}+a \partial_{t_{1}}+b, \quad B=-\frac{\tau(r+1)}{\tau(r)}, \quad C=\frac{\tau(r)}{\tau(r+1)},
$$

where $a$ and $b$ are the same quantities as shown in (5.26). $A_{n}, B_{n}, C_{n}$ are given by

$$
\begin{array}{ll}
A_{n}=\left(W_{1} \partial_{t_{1}}^{n} V_{2}^{*}+V_{1}\left(-\partial_{t_{1}}\right)^{n} W_{2}^{*}\right)_{\geq 0}, & B_{n}=-\left(W_{1} \partial_{t_{1}}^{n} V_{1}^{*}+V_{1}\left(-\partial_{t_{1}}\right)^{n} W_{1}^{*}\right)_{\geq 0}, \\
C_{n}=\left(W_{2} \partial_{t_{1}}^{n} V_{2}^{*}+V_{2}\left(-\partial_{t_{1}}\right)^{n} W_{2}^{*}\right)_{\geq 0}, & D_{n}=-\left(W_{2} \partial_{t_{1}}^{n} V_{1}^{*}+V_{2}\left(-\partial_{t_{1}}\right)^{n} W_{1}^{*}\right)_{\geq 0},
\end{array}
$$

where ()$_{\geq 0}$ stands for the projection onto nonnegative powers of $\partial_{t_{1}}$. These operators satisfy the algebraic relations

$$
A_{n}^{*}=-D_{n}, \quad B_{n}^{*}=B_{n}, \quad C_{n}^{*}=C_{n}, \quad D_{n}^{*}=-A_{n},
$$

which may be thought of as Lie algebraic counterparts of the constraint (A.4) for the dressing operators. This algebraic structure is generalized by Kac and van de Leur [3] to multicomponent hierarchies. Let us mention that these algebraic relations among $A_{n}, B_{n}, C_{n}, D_{n}$ are also derived by Kakei [9] in a inverse scattering formalism.

The evolution equations (A.5) and (A.6) can be readily converted to the evolution equations

$$
e^{\partial_{r}}\left(\begin{array}{ll}
\Psi_{1} & \Psi_{1}^{*} \\
\Psi_{2} & \Psi_{2}^{*}
\end{array}\right)=\left(\begin{array}{cc}
A & B \\
C & 0
\end{array}\right)\left(\begin{array}{ll}
\Psi_{1} & \Psi_{1}^{*} \\
\Psi_{2} & \Psi_{2}^{*}
\end{array}\right)
$$

and

$$
\partial_{t_{n}}\left(\begin{array}{ll}
\Psi_{1} & \Psi_{1}^{*} \\
\Psi_{2} & \Psi_{2}^{*}
\end{array}\right)=\left(\begin{array}{cc}
A_{n} & B_{n} \\
C_{n} & D_{n}
\end{array}\right)\left(\begin{array}{cc}
\Psi_{1} & \Psi_{1}^{*} \\
\Psi_{2} & \Psi_{2}^{*}
\end{array}\right)
$$

for the wave functions.

\section{Acknowledgements}

The author is grateful to Ralf Willox and Saburo Kakei for useful information and comments. This work is partly supported by Grant-in-Aid for Scientific Research No. 19540179 and No. 21540218 from the Japan Society for the Promotion of Science.

\section{References}

[1] Takasaki K., Differential Fay identities and auxiliary linear problem of integrable hierarchies, arXiv:0710.5356.

[2] Jimbo M., Miwa T., Soliton equations and infinite dimensional Lie algebras, Publ. Res. Inst. Math. Sci. 19 (1983), 943-1001.

[3] Kac V., van de Leur J., The geometry of spinors and the multicomponent BKP and DKP hierarchies, in The Bispectral Problem (Montreal, PQ, 1997), CRM Proc. Lecture Notes, Vol. 14, Amer. Math. Soc., Providence, RI, 1998, 159-202, solv-int/9706006.

[4] Hirota R., Ohta Y., Hierarchies of coupled soliton equations. I, J. Phys. Soc. Japan 60 (1991), 798-809.

[5] Adler M., Horozov E., van Moerbeke P., The Pfaff lattice and skew-orthogonal polynomials, Internat. Math. Res. Notices 1999 (1999), no. 11, 569-588, solv-int/9903005.

[6] Adler M., Shiota T., van Moerbeke P., Pfaff $\tau$-functions, Math. Ann. 322 (2002), 423-476, solv-int/9909010.

[7] Adler M., van Moerbeke P., Toda versus Pfaff lattice and related polynomials, Duke Math. J. 112 (2002), $1-58$. 
[8] Kakei S., Orthogonal and symplectic matrix integrals and coupled KP hierarchy, J. Phys. Soc. Japan 99 (1999), 2875-2877, solv-int/9909023.

[9] Kakei S., Dressing method and the coupled KP hierarchy, Phys. Lett. A 264 (2000), 449-458, solvint/9909024.

[10] van de Leur J., Matrix integrals and the geometry of spinors, J. Nonlinear Math. Phys. 8 (2001), 288-310, solv-int/9909028.

[11] Adler M., van Moerbeke P., Hermitian, symmetric and symplectic random ensembles: PDEs for the distribution of the spectrum, Ann. of Math. (2) 153 (2001), 149-189, math-ph/0009001.

[12] Adler M., Kuznetsov V.B., van Moerbeke P., Rational solutions to the Pfaff lattice and Jack polynomials, Ergodic Theory Dynam. Systems 22 (2002), 1365-1405, nlin.SI/0202037.

[13] Isojima S., Willox R., Satsuma J., On various solutions of the coupled KP equation, J. Phys. A: Math. Gen. 35 (2002), 6893-6909.

[14] Isojima S., Willox R., Satsuma J., Spider-web solutions of the coupled KP equation, J. Phys. A: Math. Gen. 36 (2003), 9533-9552.

[15] Kodama Y., Maruno K.-I., N-soliton solutions to the DKP hierarchy and the Weyl group actions, J. Phys. A: Math. Gen. 39 (2006), 4063-4086, nlin.SI/0602031.

[16] Kodama Y., Pierce V.U., Geometry of the Pfaff lattice, arXiv:0705.0510.

[17] Willox R., On a coupled Toda lattice and its reductions as derived from the coupled KP hierarchy, Proceedings of the Research Institute for Applied Mechanics, Kyushu University 13ME-S4 (2002), 18-23 (in Japanese).

[18] Willox R., On a generalized Tzitzeica equation, Glasgow Math. J. 47 (2005), 221-231.

[19] Santini P.M., Nieszporski M., Doliwa A., An integrable generalization of the Toda law to the square lattice, Phys. Rev. E 70 (2004), 056615, 6 pages, nlin.SI/0409050.

[20] Hu X.-B., Li C.-X., Nimmo J.J.C., Yu G.-F., An integrable symmetric $(2+1)$-dimensional Lotka-Volterra equation and a family of its solutions, J. Phys. A: Math. Gen. 38 (2005), 195-204.

[21] Gilson C.R., Nimmo J.J.C., The relation between a 2D Lotka-Volterra equation and a 2D Toda lattice, J. Nonlinear Math. Phys. 12 (2005), suppl. 2, 169-179.

[22] Zabrodin A.V., Dispersionless limit of Hirota equations in some problems of complex analysis, Theoret. and Math. Phys. 129 (2001), 1511-1525, math.CV/0104169.

[23] Teo L.-P., Fay-like identities of the Toda lattice hierarchy and its dispersionless limit, Rev. Math. Phys. 18 (2006), 1055-1073, nlin.SI/0606059.

[24] Kostov I.K., Krichever I., Mineev-Weinstein M., Wiegmann P.B., Zabrodin A., $\tau$-function for analytic curve, in Random Matrices and Their Applications, Editors P. Bleher and A. Its, Math. Sci. Res. Inst. Publ., Vol. 40, Cambridge University Press, Cambridge, 2001, 285-299, hep-th/0005259.

[25] Boyarsky A., Marshakov A., Ruchayskiy O., Wiegmann P., Zabrodin A., Associativity equations in dispersionless integrable hierarchies, Phys. Lett. B 515 (2001), 483-492, hep-th/0105260.

[26] Teo L.-P., Analytic functions and integrable hierarchies - characterization of tau functions, Lett. Math. Phys. 64 (2003), 75-92, hep-th/0305005.

[27] Kodama Y., Pierce V.U., Combinatorics of dispersionless integrable systems and universality in random matrix theory, arXiv:0811.0351.

[28] Konopelchenko B., Martinez Alonso L., Integrable quasi-classical deformations of algebraic curves, J. Phys. A: Math. Gen. 37 (2004), 7859-7877, nlin.SI/0403052.

[29] Kodama Y., Konopelchenko B., Martinez Alonso L., Integrable deformations of algebraic curves, Theoret. and Math. Phys. 144 (2005), 961-967.

[30] Date E., Kashiwara M., Jimbo M., Miwa T., Transformation groups for soliton equations, in Nonlinear Integrable Systems - Classical Theory and Quantum Theory (Kyoto, 1981), World Scientific Publishing, Singapor, 1983, 39-119.

[31] Dickey L., Soliton equations and Hamiltonian systems, Advanced Series in Mathematical Physics, Vol. 12, World Scientific Publishing Co., Inc., River Edge, NJ, 1991.

[32] Kac V.G., van de Leur J.W., The $n$-component KP hierarchy and representation theory, in Important Developments in Soliton Theory, Springer Ser. Nonlinear Dynam., Springer, Berlin, 1993, 302-343, hep-th/9308137.

[33] Takasaki K., Takebe T., Integrable hierarchies and dispersionless limit, Rev. Math. Phys. 7 (1995), 743-808, hep-th/9405096. 